\title{
Tuning mechanical performance of poly(ethylene glycol) and agarose interpenetrating network hydrogels for cartilage tissue engineering
}

\author{
DA Rennerfeldt ${ }^{1}$, AN Renth ${ }^{2}$, Z Talata $^{3}$, SH Gehrke ${ }^{1}$, and MS Detamore ${ }^{1}$ \\ ${ }^{1}$ Department of Chemical and Petroleum Engineering, University of Kansas, Lawrence, Kansas \\ 66045 \\ 2Bioengineering Program, University of Kansas, Lawrence, KS 66045 \\ ${ }^{3}$ Department of Mathematics, University of Kansas, Lawrence, KS 66045
}

\section{Abstract}

\begin{abstract}
Hydrogels are attractive for tissue engineering applications due to their incredible versatility, but they can be limited in cartilage tissue engineering applications due to inadequate mechanical performance. In an effort to address this limitation, our team previously reported the drastic improvement in the mechanical performance of interpenetrating networks (IPNs) of poly(ethylene glycol) diacrylate (PEG-DA) and agarose relative to pure PEG-DA and agarose networks. The goal of the current study was specifically to determine the relative importance of PEG-DA concentration, agarose concentration, and PEG-DA molecular weight in controlling mechanical performance, swelling characteristics, and network parameters. IPNs consistently had compressive and shear moduli greater than the additive sum of either single network when compared to pure PEG-DA gels with a similar PEG-DA content. IPNs withstood a maximum stress of up to $4.0 \mathrm{MPa}$ in unconfined compression, with increased $P E G$-DA molecular weight being the greatest contributing factor to improved failure properties. However, aside from failure properties, $P E G$ $D A$ concentration was the most influential factor for the large majority of properties. Increasing the agarose and PEG-DA concentrations as well as the PEG-DA molecular weight of agarose/ PEG-DA IPNs and pure PEG-DA gels improved moduli and maximum stresses by as much as an order of magnitude or greater compared to pure PEG-DA gels in our previous studies. Although the viability of encapsulated chondrocytes was not significantly affected by IPN formulation, glycosaminoglycan (GAG) content was significantly influenced, with a 12-fold increase over a three-week period in gels with a lower PEG-DA concentration. These results suggest that mechanical performance of IPNs may be tuned with partial but not complete independence from biological performance of encapsulated cells.
\end{abstract}

\section{Keywords}

Cartilage; PEG-DA; Agarose; Interpenetrating Networks; Hydrogels; Mechanical Performance

\footnotetext{
(C) 2013 Elsevier Ltd. All rights reserved.

Corresponding Author: Michael S. Detamore, University of Kansas, Department of Chemical and Petroleum Engineering, 4132 Learned Hall, 1530 W 15 ${ }^{\text {th }}$ St, Lawrence, KS 66045, Phone: (785) 864-4943, FAX: (785) 864-4967, detamore@ku.edu.

Publisher's Disclaimer: This is a PDF file of an unedited manuscript that has been accepted for publication. As a service to our customers we are providing this early version of the manuscript. The manuscript will undergo copyediting, typesetting, and review of the resulting proof before it is published in its final citable form. Please note that during the production process errors may be discovered which could affect the content, and all legal disclaimers that apply to the journal pertain.
} 


\section{Introduction}

Mechanical integrity is an important property of hydrogels used for cartilage regeneration and is often a major barrier in usability. Progress in improving hydrogel mechanical strength was significantly advanced with the development of a special class of interpenetrating network (IPN) hydrogels with superior mechanical properties (termed "double-network hydrogels") by the research team of Gong and Osada at Hokkaido University (Japan) [1, 2]. An IPN is synthesized by constructing a single-network hydrogel (first network), soaking the gel in a monomer solution of the second component long enough to equilibrate, and then photopolymerizing the second network that interlocks with the first. While IPN formation typically enhances the performance of hydrogels in biomedical applications, conventional IPNs do not show more than additive improvement in mechanical integrity over either individual network $[1,3]$. However, double-network (DN) hydrogels exhibit mechanical properties sometimes orders of magnitude greater than their individual components [2, 4].

Since the development of the DN hydrogel in 2003, Gong and Osada have recognized the comparability of DN properties to native cartilage and have consequentially studied their potential for cartilage replacement $[5,6]$, with other research groups following suit $[7,8]$. More recently, they developed a method using a DN hydrogel to attempt in vivo articular cartilage regeneration without the use of encapsulated cells [9]. Other studies have led to extremely strong DN hydrogels $[10,11]$, though without the specific aim of cartilage repair or regeneration. Most methods for synthesizing DN hydrogels are not compatible for cell encapsulation, and there have been very few attempts to produce cell-laden DN gels. Recently, Tang and coworkers [12] reported the encapsulation of chondrocytes into a DN hydrogel made of gellan gum and carboxymethyl chitosan to evaluate the material's biocompatibility. Mechanical testing was also done on acellular gels, with their stiffest gel showing a compressive modulus of $\sim 600 \mathrm{kPa}$, which is relatively high for a cellularly-viable gel. However, this gel permanently deformed upon compression, raising the concern for recovery for this particular formulation. Shin and coworkers [13] recently encapsulated fibroblasts into gelatin/gellan gum DN hydrogels to evaluate their cytocompatibility and mechanical properties. They reported an acellular IPN that could withstand a maximum compression of $\sim 7 \mathrm{MPa}$ and had a compressive modulus of $\sim 110 \mathrm{kPa}$. While both studies showed cells could survive the encapsulation process, viability was not tested beyond 1 week for Tang and coworkers and 3 days for Shin and coworkers.

Network parameters such as swelling degree and crosslinking density, which are important in determining whether the DN hydrogels provide a microenvironment conducive to waste/ nutrient transport, have been reported for single-network hydrogels, particularly to determine their relationship with proteoglycan distribution [14]. However, the equations for calculating these parameters have not yet been applied to hydrogels with more than one network for cartilage regeneration. Furthermore, because the role of cell encapsulation is to stimulate regrowth of the patient's own cartilage, it is also important to evaluate not only the viability of encapsulated cells over a longer period of time but also whether the cells are healthy and producing ECM. Additionally, reporting the fracture properties of hydrogels meant for load-bearing tissue applications is just as essential as reporting mechanical stiffness. Finally, encapsulated cells that produce ECM while degrading or metabolizing the gel could potentially affect the gel's mechanical properties, so mechanical testing on cellladen hydrogels is also crucial in determining whether the material is truly appropriate in providing mechanical compensation in vivo.

Our group has recently shown that chondrocytes can survive the encapsulation process in an IPN consisting of $2 \% \mathrm{w} / \mathrm{v}$ agarose and $15 \% \mathrm{w} / \mathrm{v}$ poly(ethylene glycol) diacrylate (PEG-DA, $\mathrm{MW}=2000 \mathrm{Da})[15,16]$. Many agarose studies have shown a high degree of success for 
cell viability and GAG production, though these constructs are severely limited by their inferior mechanical performance. PEG-DA, a synthetic, hydrophilic polymer, has also been widely studied for cartilage regeneration and has mechanical properties far exceeding those of agarose. Nguyen and coworkers [17] recently released an extensive overview of the use of PEG-DA for cartilage tissue engineering, including the concentrations, molecular weights, methods, mechanical properties tested, and results of several research groups who have studied PEG-based hydrogels.

While our previous studies of agarose/PEG-DA IPNs showed a large improvement in mechanical performance compared to both single networks, we hypothesized that further mechanical improvements would be observed by increasing the concentrations of both networks as well as the molecular weight of PEG-DA while still maintaining cell viability. Three PEG-DA concentrations (10\%, 15\%, and $20 \% \mathrm{w} / \mathrm{v})$, three agarose concentrations ("0\%" for pure PEG-DA hydrogels, $2 \%$ and $5 \% \mathrm{w} / \mathrm{v}$ for IPNs), and three PEG-DA molecular weights $(2000,3400$, and $6000 \mathrm{Da})$ were studied in a full-factorial design for a total of 27 formulations. Our objective was to determine which of these three factors most significantly contributed to differences in mechanical properties, network parameters, and swelling characteristics. Of these 27 formulations, nine representative groups were selected for encapsulation to examine viability as well as the mechanical properties of cell-laden scaffolds compared to their acellular counterparts, and three were further selected for biochemical analysis.

\section{Materials and Methods}

\subsection{Materials}

High-purity PEG-DA (molecular weights 2000, 3400, and 6000 Da; purity >99\%) was purchased from SunBio (Anyang City, South Korea). 2-Hydroxyethyl agarose (Type VII) was purchased from Sigma-Aldrich (St. Louis, MO, USA). The photoinitiator Irgacure 2959 (I-2959) was acquired from Ciba (Basel, Switzerland).

\subsection{Agarose network synthesis}

Agarose powder was added to phosphate-buffered saline (PBS, $0.01 \mathrm{M}$ ) to yield 2\% and 5\% $\mathrm{w} / \mathrm{v}$ solutions and autoclaved for 30 minutes. Solutions were then pipetted into cylindrical silicon rubber molds ( $\sim 5 \mathrm{~mm}$ diameter, $\sim 2 \mathrm{~mm}$ height), pressed between glass plates, and cooled at $4{ }^{\circ} \mathrm{C}$ for at least 10 minutes. Gels were then placed in PBS to equilibrate for at least 24 hours before use.

\subsection{PEG-DA network synthesis}

Irgacure 2959 was added to a 70/30 mixture of ethanol and deionized (DI) water to create a $10 \% \mathrm{w} / \mathrm{v}$ photoinitiator solution. $10 \%, 15 \%$, and $20 \% \mathrm{w} / \mathrm{v}$ solutions of PEG-DA (molecular weight 2000,3400, and $6000 \mathrm{Da}$ each) in PBS were prepared, and $10 \mu \mathrm{L}$ of photoinitiator solution was added to each $\mathrm{mL}$ of PEG-DA solutions. The solutions were then placed in rectangular silicon rubber molds ( $\sim 2 \mathrm{~mm}$ height), pressed between optical glass microscope slides, and exposed to $312 \mathrm{~nm}$ light $\left(3.0 \mathrm{~mW} / \mathrm{m}^{2}\right.$, XL-1000, Spectronics Corp.; Lincoln, NE) for five minutes on each side. Gel samples were cut using a $3 \mathrm{~mm}$ biopsy punch, and gels were transferred to PBS to equilibrate for at least 24 hours before use.

\subsection{IPN formation}

Four cylindrical agarose gels were added for each $\mathrm{mL}$ of PEG-DA/photoinitiator solution and soaked under constant agitation. The length of the soaking times needed for adequate diffusion were calculated based on literature data [18] and were dependent on the PEG-DA molecular weight $(2,3.5$, and 6 hours for molecular weights of 2000,3400, and 6000, 
respectively). The agarose gels were then placed in rectangular molds ( $2 \mathrm{~mm}$ height) between two optical glass microscope slides and exposed to $312 \mathrm{~nm}$ light, $3.0 \mathrm{~mW} / \mathrm{m}^{2}$ (XL-1000, Spectronics Corp.) for 5 minutes on each side. Samples were then cut using a 3 $\mathrm{mm}$ biopsy punch and added to PBS to equilibrate for at least 24 hours before use. The formulations are reported according to the following structure: agarose concentration/PEGDA concentration (PEG-DA molecular weight). As an example, the formulation 2\%/15\% (2k) is an IPN with $2 \%$ w/v agarose soaked in a 15\% w/v PEG-DA (2000 Da molecular weight) monomer solution prior to photopolymerization.

\subsection{Chondrocyte isolation}

10 ankles of 5 juvenile Chester White hogs (female, $\sim 3$ months old, $~ 120 \mathrm{lbs}$ ) were obtained from Winchester Meat Processing, Inc. (Winchester, KS). Cells were harvested within 24 hours after slaughter following methods described in our previous publications $[19,20]$. The articular cartilage was removed manually with a scalpel and minced under sterile conditions. The cartilage was then digested in a $2 \mathrm{mg} / \mathrm{mL}$ sterile-filtered solution of type II collagenase (300 U/mg, Worthington Biochemical; Lakewood, NJ) for 24 hours at $37^{\circ} \mathrm{C}$. Cells were then filtered from their ECM, centrifuged, and resuspended in culture medium consisting of Dulbecco's modified Eagle medium with $4.5 \mathrm{~g} / \mathrm{L}$ D-glucose, $10 \%$ fetal bovine serum (FBS), $1 \%$ nonessential amino acids, $1 \%$ sodium pyruvate, and $1 \%$ penicillin-streptomycin fungicide. Cells were fed every other day with the supplemented medium until $\sim 90 \%$ confluent. The medium and supplements were obtained from Invitrogen (Grand Island, NY). Cells were not pooled until after the first passage, during the encapsulation process.

\subsection{Cell encapsulation}

2.6.1 Encapsulation into IPN gels-Two solutions of agarose (3\% and $7.5 \% \mathrm{w} / \mathrm{v})$ in PBS were prepared and autoclaved for 30 minutes. Meanwhile, cells were detached from their flasks with trypsin-ethylenediaminetetra acetic acid and labeled as passage 1 (P1). At this point, $\mathrm{P} 1$ cells were pooled and resuspended in PBS at 30 million cells $/ \mathrm{mL}$ to begin the encapsulation procedure. Once cooled to $39^{\circ} \mathrm{C}$, the cell suspension was added to each solution of molten agarose in a 1:2 ratio to produce $2 \%$ and $5 \%$ agarose solutions with a seeding density of 10 million cells $/ \mathrm{mL}$. Each solution was pipetted into sterilized silicon rubber molds ( $\sim 5 \mathrm{~mm}$ diameter, $\sim 2 \mathrm{~mm}$ height), pressed between two glass plates, and cooled at $4{ }^{\circ} \mathrm{C}$ for 10 minutes. The cell-encapsulated gels were then added to untreated 24well plates (Becton Dickenson; Franklin Lakes, NJ), supplied with $1.5 \mathrm{~mL}$ growth medium, and placed in a sterile $37^{\circ} \mathrm{C}$ incubator. After 24 hours, gels were added to sterile-filtered solutions of PEG-DA (10\%, 15\%, and 20\% w/v; 2000, 3400, and 6000 Da molecular weights) with $0.1 \%$ Irgacure photoinitiator in PBS. Formulations selected for encapsulation are presented in Table 1 . Gels were left to soak at $37^{\circ} \mathrm{C}$ and constant agitation for diffusion times dependent on the molecular weight of PEG-DA (2, 3.5, and 6 hours for molecular weights of 2000, 3400, and 6000, respectively). Afterward, gels were placed in sterilized rectangular silicon molds between sterilized optical glass microscope slides and exposed for 5 minutes on each side to $312 \mathrm{~nm}$ light $\left(3.0 \mathrm{~mW} / \mathrm{cm}^{2}\right)$. Gel samples were then cut using a 3 $\mathrm{mm}$ biopsy punch and returned to growth media for 24 hours before analysis.

2.6.2 Encapsulation into PEG-DA gels-Sterile filtered solutions of PEG-DA (30\% w/ v, 2000, 3400, and 6000 Da molecular weights) containing $0.1 \%$ photoinitiator were combined under sterile conditions in a 2:1 ratio with cells suspended in PBS (30 million cells $/ \mathrm{mL}$ ), yielding three solutions of $20 \% \mathrm{w} / \mathrm{v}$ PEG-DA with a seeding density of 10 million cells $/ \mathrm{mL}$. Solutions were placed in autoclaved rectangular molds between two optical glass microscope slides and exposed to $312 \mathrm{~nm}$ light $\left(3.0 \mathrm{~mW} / \mathrm{cm}^{2}\right)$ for five minutes on each side. A $3 \mathrm{~mm}$ biopsy punch was used to cut samples, which were then placed in growth media for 24 hours prior to analysis. 


\subsection{GAG and DNA content analysis}

Three of the original 27 formulations (indicated on Table 1, n=4) were analyzed for ECM production at 0 and 3 weeks following methods recently described [16]. These formulations were chosen based on the mechanical performance of their acellular counterparts. DNA content was determined with a Picogreen assay (Molecular Probes; Eugene, OR), which was used according to the manufacturer's directions. GAG content was quantified using a dimethylmethylene blue assay (Biocolor; Carrickfergus, Northern Ireland) and normalized to DNA content.

\subsection{Cell viability analysis}

A live/dead viability cytotoxicity kit (Invitrogen) containing $4 \mathrm{mM}$ calcein AM (to stain live cells) and $2 \mathrm{mM}$ ethidium homodimer-1 (to stain dead cells) was used at 24 hours, 1 week, and 3 weeks to assess the cell viability of chondrocytes encapsulated in IPNs and PEG-DA gels. Cylindrical constructs $(n=3)$ were sectioned parallel to the circular face and incubated at $37{ }^{\circ} \mathrm{C}$ in the live/dead reagents for 45 minutes before imaging. At the 24 hour (week 0) time point, images were captured using an Eclipse TS100-F inverted microscope (Nikon Instruments Inc.; Melville, NY) with QCapture Pro software (version 5.1). At weeks 1 and 3 , Z-scans were performed to the maximum resolution depth $(350-500 \mu \mathrm{m})$ in areas representative of the overall gels using a Yokugawa CSU10 spinning disk attached to an Olympus IX 81 microscope with $561 \mathrm{~nm}$ excitation/585LP emission and $488 \mathrm{~nm}$ excitation/ 515-540 nm emission filters. CellProfiler and CellProfiler Analyst software (Broad Institute; Cambridge, MA) was used to analyze the images and determine the percent of total cells viable at all three time points.

\subsection{Mechanical analysis}

Gel diameter was measured with a micrometer under a standard stereomicroscope (approximately 10x magnification). Samples were then loaded onto an RSA-III dynamic mechanical analyzer (TA Instruments; New Castle, DE). Samples were compressed unconstrained to $95 \%$ of their original height or to fracture, which was measured directly with the RSA-III. A compression rate of $0.005 \mathrm{~mm} / \mathrm{s}$, corresponding to an average of $15 \%$ / min, was used. The compressive modulus (E) was calculated as the slope up to an $\mathrm{x}$-axis value of $10 \%$ strain of the stress versus strain curve. Using the neo-Hookean model for ideal elastomers, the shear modulus $(\mathrm{G})$ was calculated as the slope of a plot of the stress versus $\left(\lambda-1 / \lambda^{2}\right)$, where $\lambda=\mathrm{L} / \mathrm{L}_{0}, \mathrm{~L}=$ strained thickness, and $\mathrm{L}_{0}=$ original thickness [21], up to a $(\lambda-1 /$ $\left.\lambda^{2}\right)$ value of 10 or to fracture.

\subsection{Solids content characterization}

Gels were created using the methods described above. Once crosslinked, gels were placed in excess DI water for at least 24 hours, then dried for at least 72 hours in a desiccation chamber. Each sample was weighed a total of three times using a high precision balance (Shimadzu Corporation; Kyoto, Japan): once immediately after crosslinking $\left(\mathrm{m}_{\mathrm{r}}\right)$, once after swelling $\left(\mathrm{m}_{\mathrm{s}}\right)$, and again after drying $\left(\mathrm{m}_{\mathrm{d}}\right)$.

The dry mass $\left(\mathrm{m}_{\mathrm{d}}\right)$ and swollen mass $\left(\mathrm{m}_{\mathrm{s}}\right)$ of the PEG-DA gels and IPNs $(\mathrm{n}=4)$ were used to calculate the mass swelling ratio $(\mathrm{q})$ :

$$
\mathrm{q}=\frac{\mathrm{m}_{\mathrm{s}}}{\mathrm{m}_{\mathrm{d}}}
$$

The solid fraction, $\mathrm{q}^{-1}$, was multiplied by 100 to give the total solids percentage of each gel. The conversion of PEG-DA in the single network (SN) gels was then calculated as the PEGDA dry mass divided by the theoretical mass of PEG-DA in the sample before crosslinking: 
where $\varphi_{0}$ is the original $\mathrm{w} / \mathrm{v}$ fraction of monomer solution $(0.10,0.15$, and 0.20$)$, and $\mathrm{V}$ is the calculated volume of the sample based on the mold thickness and biopsy punch diameter. To estimate the conversion of PEG-DA in the IPNs, solids content characterization was also performed on $2 \%$ and $5 \% \mathrm{w} / \mathrm{v}$ agarose gels $(\mathrm{n}=10)$ for use in the following equation:

$$
\text { \%Conversion of PEG-DA in IPNs }=\frac{\mathrm{m}_{\mathrm{d}}(\mathrm{IPN})-\overline{\mathrm{m}}_{\mathrm{d}}(\text { agarose })}{\kappa \phi_{0} \mathrm{~V}} \times 100 \%
$$

where $\mathrm{K}$ is the partition coefficient (assumed to be 1, though can be lower), the numerator is the estimated PEG-DA dry mass in the IPN, and the overbar denotes an average value.

Similarly, the total solids percentage of PEG-DA in IPNs was calculated by subtracting the average total solids of agarose from the total solids of the IPNs.

\subsection{Network Parameters}

The equilibrium volume swelling ratio $(\mathrm{Q})$ was calculated using polymer and solvent densities[22] ( $\rho_{\mathrm{p}}$ and $\rho_{\mathrm{s}}$, respectively), where $\rho_{\mathrm{p}}$ for PEG is taken as $1.12 \mathrm{~g} / \mathrm{mL}$ [23] and $\rho_{s}$ for water was assumed to be $1 \mathrm{~g} / \mathrm{mL}$ :

$$
\mathrm{Q}=1+\frac{\rho_{\mathrm{p}}}{\rho_{\mathrm{s}}}\left(\frac{\mathrm{m}_{\mathrm{s}}}{\mathrm{m}_{\mathrm{d}}}-1\right)
$$

The following equation, assuming affine network chain deformation [24], was used to determine the effective crosslink density $\left(\rho_{\mathrm{x}}\right)$ :

$$
\rho_{\mathrm{x}}=\frac{\mathrm{G}}{\mathrm{RT} \phi_{2, \mathrm{~s}}^{1 / 3} \phi_{2, \mathrm{r}}^{2 / 3}}
$$

where $\mathrm{G}$ is the shear modulus, $\mathrm{R}$ is the gas constant, $\mathrm{T}$ is the absolute temperature, $\varphi_{2, \mathrm{~s}}$ is the polymer volume fraction $\left(\mathrm{Q}^{-1}\right)$, and $\varphi_{2, \mathrm{r}}$ is the polymer volume fraction at network formation. Similar to $\varphi_{2, \mathrm{~s}}, \varphi_{2, \mathrm{r}}$ was calculated taking the mathematical inverse of Equation 4, replacing $\mathrm{m}_{\mathrm{s}}$ with the mass of the gel after crosslinking but before swelling $\left(\mathrm{m}_{\mathrm{r}}\right)$.

The molecular weight between crosslinks $\left(\mathrm{M}_{\mathrm{c}}\right)$ was calculated using Equation 6:

$$
\mathrm{M}_{\mathrm{c}}=\frac{\rho_{\mathrm{p}}}{\rho_{\mathrm{x}}}
$$

The polymer-solvent interaction parameter $(\mathrm{X})$ was also calculated based on the affine model as follows [21]:

$$
\chi=\frac{-\ln \left(1-\phi_{2, \mathrm{~s}}\right)-\phi_{2, \mathrm{~s}}-\tilde{\mathrm{V}}_{1} \rho_{\mathrm{x}} \phi_{2, \mathrm{r}}\left[\left(\frac{\phi_{2, \mathrm{~s}}}{\phi_{2, \mathrm{~s}}}\right)^{1 / 3}-\frac{1}{2}\left(\frac{\phi_{2, \mathrm{~s}}}{\phi_{2, \mathrm{~s}}}\right)\right]}{\phi_{2, \mathrm{~s}}^{2}}
$$

${ }_{1}$ is the solvent molar volume $(18 \mathrm{~g} / \mathrm{mol})$.

Assuming the active network chains are composed of PEG [25-27], a mesh size ( $)$ ) for the PEG-DA network was calculated using methods previously described by the widely-used equation: 


$$
\xi=\phi_{2, \mathrm{~s}}^{-1 / 3} \mathrm{C}_{\mathrm{n}}^{1 / 2} \mathrm{n}^{1 / 2} 1
$$

where $\mathrm{C}_{\mathrm{n}}$ is the characteristic ratio of the polymer (taken to be 4 for PEG) [27], $I$ is the weighted average of the $\mathrm{C}-\mathrm{C}$ and $\mathrm{C}-\mathrm{O}$ backbone bond lengths $(1.47 \AA$ ), and $\mathrm{n}$ is the number of bonds between crosslinks, calculated as:

$$
\mathrm{n}=\frac{3 \mathrm{M}_{\mathrm{c}}}{\mathrm{M}_{\mathrm{r}}}
$$

The molecular weight of the repeating unit $\left(\mathrm{M}_{\mathrm{r}}\right)$ used was $44 \mathrm{~g} / \mathrm{mol}$ for PEG [27], and $\mathrm{M}_{\mathrm{c}}$ was calculated for each gel using Equation 6.

The above equations have been widely used to characterize network structures [14, 28]. However, it is important to recognize that $\rho_{\mathrm{x}}, \mathrm{M}_{\mathrm{c}}, \xi$ and $\xi$ are all empirical parameters whose values depend upon the model used to calculate them. Theoretically, crosslink density is the number of elastically effective junctions per unit volume, but it is well-recognized that this cannot be correlated directly with the number of crosslinking reactions because not all such reactions lead to elastically effective junctions, nor does it account for chain entanglement effects [29]. Furthermore, arguments have been made that a phantom network better approximates hydrogel network parameters than the affine model used here [30], which would cause $\rho_{\mathrm{x}}$ to differ by a factor of two from the calculation based on the affine model (Eq. 5). Flory-Rehner theory, as cited in Equation 8, is limited both by the validity of the elasticity model used as well as the solution thermodynamics model, which yields $X-a$ model known not to be especially accurate for hydrophilic polymers [21]. Additionally, the equation for mesh size originally proposed by Peppas and Barr-Howell [31] assumes a highly idealized network structure of uniformly crosslinked chains with a uniform distribution of chains throughout the volume of the gel, while real gels are hetereogenous with a variety of length scales that contribute in different ways to mechanical properties, swelling degree, solute diffusivities and so forth [21]. Additionally, a wide variety of methods are used to estimate $\mathrm{Q}$ (and therefore $\varphi_{2, \mathrm{~s}}$ ) [16, 22, 24- 27, 32-34].

Nevertheless, the parameters $\rho_{\mathrm{x}}, \mathrm{M}_{\mathrm{c}}, \mathrm{X}$ and $\xi$ as calculated here are widely used to characterize gels and for the purposes of this study help understand which hydrogel factors (monomer concentrations and PEG-DA molecular weight) most contribute to their properties, however approximated. For example, crosslink density $\left(\rho_{\mathrm{x}}\right)$ accounts for the effect of swelling on the modulus and thus provides a better basis for comparison of network structures than by considering modulus alone. The molecular weight between crosslinks is essentially a redefinition of the crosslink density whose value is more easily related to the polymer network structure of different gel types, even when recognized as a simple correlating parameter. The Flory-Huggins $X$ parameter is widely reported for different polymers, and its comparison against previously reported values provides a useful test for the consistency of our work with others. Finally, even though mesh size is only a model parameter, its calculation helps with interpretation of how changes in swelling affect molecular structure of different networks because it incorporates information about chain conformation, which is not otherwise considered in Flory-Rehner theory for swelling.

\subsection{Statistical Analysis}

For statistical inference in Sections 4.1 and 4.2, a three-factor analysis of variance (ANOVA), followed by a Tukey's Honestly Significant Difference post hoc test, was performed with SPSS 20.0 (IBM; Armonk, NY) on the 27 acellular experimental groups. The test had balanced design, as four observations were taken for each group. The model included the three factors (PEG-DA concentration, agarose concentration, and PEG-DA 
molecular weight) and the possible interactions between them. The results were then plotted for further analysis (Figures 1, 3, 5, and 6), and based on the post hoc tests, each group was marked if it significantly differed from the other groups varying only in one factor. For each property, the plots showed which factor had the most significant effect. Such a claim was also supported by the ratio of the sum of squares belonging to the most influential factor compared to the total sum of squares, which included the contribution of not only the factors but the interactions also. We reported this ratio (termed "SS ratio" from here forward) to indicate the significance of a factor as well. SPSS was also used to construct standard box plots for outlier elimination. In Section 3.3 and 3.4, the statistical inference required only a single-factor analysis of variance, followed by a Tukey's Honestly Significant Difference post hoc test.

\section{Results}

\subsection{Mechanical Analysis (Acellular)}

The maximum stress and maximum strain percent of all 27 formulations are displayed in Figure 1. Some formulations did not fracture until reaching a stress of 3 to $4 \mathrm{MPa}$. A few samples from one formulation, $2 \% / 20 \%$ (6k), did not fracture at all with $95 \%$ compression (Figure 2), and thus the term "maximum stress" was used rather than "fracture stress." It has been shown in previous studies $[15,16]$ that stress-strain curves for agarose/PEG-DA IPNs show a sharp increase in slope beyond 50\% strain, resulting in large standard deviations in maximum stress for moderate standard deviations in maximum strain. Increasing PEG-DA and agarose concentrations showed improvement in the amount of stress the gels could withstand before failure, though few statistically significant differences were found among groups, due in part to large standard deviations. Table 2 presents the most significant contributing factor for each property as well as the relationship between the two. PEG-DA molecular weight was by far the greatest contributing factor to both maximum stress (SS ratio $=0.46)$ and maximum strain (SS ratio $=0.73$ ), with increases in PEG-DA leading to an increase in both properties.

Shear modulus (G) data are given in Figure 3a. PEG-DA concentration was statistically the most influential factor for the shear modulus (SS ratio $=0.58$ ) as well as for the compressive modulus ( $\mathrm{SS}$ ratio $=0.58$ ), with increases in concentration leading to increases in both elastic and shear moduli. Both moduli had a range of approximately an order of magnitude: 25 to $230 \mathrm{kPa}$ for the shear modulus and 49 to $661 \mathrm{kPa}$ for the compressive modulus. The ratio of compressive modulus (E) to shear modulus $(\mathrm{G})$ is shown in Figure 3b. Compression tests on elastic hydrogels typically give E/G ratios slightly above 3 [35], which was observed within one standard deviation for many formulations. These $\mathrm{E} / \mathrm{G}$ ratios are in agreement with our previous work on IPNs [15, 16]. Statistically, PEG-DA molecular weight was the most significantly influential factor on $\mathrm{E} / \mathrm{G}$ ratios $(\mathrm{SS}$ ratio $=0.41$ ), followed very closely by agarose concentration (SS ratio $=0.40$ ), and increases in both led to an increase in E/G. Representative neo-Hookean elasticity model plots of select formulations are presented in Figure 4 to demonstrate systematic trends in E/G ratios with respect to agarose concentration and PEG-DA molecular weight.

\subsection{Solids Content Analysis (Acellular)}

The acellular IPNs and PEG-DA gels were characterized for their swelling behavior (Figure 5a). Many significant differences in swelling were found among groups, and PEG-DA concentration was the most significant contributing factor $(\mathrm{SS}$ ratio $=0.61$ ). Increases in PEG-DA concentration led to decreases in swelling, and trends generally showed an inverse relationship between PEG-DA concentration and the shear modulus. Average mass swelling ratios for IPNs and pure PEG-DA gels ranged from 6.0 to 14.6, and pure agarose had much 
higher degrees of swelling ( $38.0 \pm 1.9$ for $2 \%$ and $21.3 \pm 1.9$ for $5 \%)$. The solids content data were used to calculate the PEG-DA apparent conversion, and agarose concentration was by far the most significant contributing factor (SS ratio $=0.75$ ). Pure PEG-DA gels had a conversion ranging from $84.5 \%$ to $90.8 \%$, while conversion in IPNs was generally much lower (an average of $67.7 \pm 4.8 \%$ for IPNs with $2 \%$ agarose and an average of $76.8 \pm 10.4 \%$ for IPNs with 5\% agarose). Additionally, the total solids content was calculated for each gel, including agarose. $2 \% \mathrm{w} / \mathrm{v}(1.96 \% \mathrm{w} / \mathrm{w})$ pure agarose gels had a solids content of $2.6 \pm$ $0.1 \%$, while $5 \% \mathrm{w} / \mathrm{v}(4.8 \% \mathrm{w} / \mathrm{w})$ pure agarose gels had a solids content of $4.7 \pm 0.5 \%$. These average values were subtracted from IPN total solids percentages to determine the total PEG-DA solids content in IPNs (Figure 5b). As with apparent conversion, PEG-DA concentration was the most significant contributing factor $(\mathrm{SS}$ ratio $=0.62)$. With the exception of one case, IPNs had a significantly lower PEG-DA content than their corresponding pure PEG-DA gels $(\mathrm{p}<0.05)$.

Data from the solids content analysis and shear modulus measurements were used to calculate network mesh size (Figure 6a). Mesh sizes varied from 5.9 to $17.0 \mathrm{~nm}$ and increased with decreasing PEG-DA concentration. Although PEG-DA concentration was statistically the most influential factor (SS ratio $=0.49$ ), mesh sizes also increased when a higher PEG-DA molecular weight was used. Crosslink density is displayed in Figure 6b. PEG-DA concentration was the factor with the most significant impact to crosslink density as well (SS ratio $=0.38$ ), however no significant differences among PEG-DA concentrations were observed in IPNs with the highest PEG-DA molecular weight.

For all 27 groups $(\mathrm{n}=4)$, the combined average polymer-solvent interaction parameter $(\mathrm{X})$ was $0.510 \pm 0.002$. Small increases were observed in the calculated polymer-solvent interaction parameters with increases in agarose and PEG-DA concentrations as well as with decreases in PEG-DA molecular weight, and overall the values ranged from 0.494 to 0.532 . Very few significant differences were observed when comparing three groups at a time for each factor. However, significant differences were observed the majority of the time between gels with PEG-DA molecular weights of 2000 and $6000 \mathrm{Da}$.

3.3 GAG Production and Cell Viability-Three formulations were selected to determine sulfated GAG composition of cell-laden gels at 0 and 3 weeks (Figure 7a). These formulations were chosen because 0\% agarose/20\% PEG-DA (2k PEG-DA molecular weight) had the highest shear modulus and the second-highest compressive modulus out of all 27 acellular formulations tested and 2\%/20\% (6k) had the highest maximum stress $(4.0$ MPa). $2 \% / 10 \%(6 \mathrm{k})$ was used as a basis of comparison for the PEG-DA concentration. The pure PEG-DA gels did not display a statistically significant change in GAG content from 0 to 3 weeks. However, the IPNs containing $20 \%$ and $10 \%$ w/v PEG-DA showed a 4-fold and 16-fold increase, respectively, in GAG content per gel from 0 to 3 weeks. DNA content analysis (Figure 7b) indicated that the DNA content was similar for all three groups at week 0 , however samples taken from the 2\%/10\% (6k) group at week 3 had $69 \%$ and $78 \%$ greater DNA content than the $2 \% / 20 \%(6 \mathrm{k})$ and $0 \% / 20 \%(2 \mathrm{k})$ groups, respectively. Therefore, the total accumulated GAG content normalized to DNA content was also determined (Figure 7c). IPNs containing 10\% PEG-DA had approximately twice the normalized GAG content as IPNs with 20\% PEG-DA, and both of the IPN groups had a higher normalized GAG content -12 -fold and 5 -fold for the $2 \% / 10 \%$ (6k) and 2\%/20\% (6k) groups, respectively at week 3 than at week 0 .

Cell viability was tested using a live/dead assay containing calcein AM (green, representing live cells) and ethidium bromide (red, representing dead cells) fluorescent markers. A preliminary experiment was first conducted to ensure that encapsulated cells could survive the longest PEG-DA diffusion time (6 hours) in the highest PEG-DA concentration (20\% w/ 
v). Figure 8 provides a representative demonstration that cells encapsulated in IPNs containing 2\% w/v agarose and 20\% w/v PEG-DA (6000 Da molecular weight) maintained high viability 24 hours after the encapsulation process despite a longer equilibration time in a higher PEG-DA concentration than previously used. Nine formulations were then selected for chondrocyte encapsulation to determine cell viability at 0,1 , and 3 weeks. Although there was a high degree of variability among samples within each group at each time point, ranging mostly from 30 to 90 percent, the majority of formulations had at least one of three samples that remained over 50\% viable three weeks after encapsulation. No statistical significance was found among groups or time points.

\subsection{Mechanical Analysis (Cell-Laden)}

Unconfined compression testing was performed on cell-laden samples 0 and 3 weeks after encapsulation. The same nine formulations were used as those selected for viability analysis. Figure 9 displays the maximum strain percents of the gels at both time points, which were compared to the measurements obtained on the previous acellular gels (Fig. 1a). For all nine cell-laden groups, the maximum strain did not change from week 0 to week 3 . However, three of the nine cell-laden formulations had a lower maximum strain percent for both time points $(\mathrm{p}<0.05)$ compared to their acellular counterparts, differing by as much as 30 strain percent. The maximum stress is presented similarly in Figure 10. As with maximum strain percent, there were no significant differences between weeks 0 and 3 for any group, however in several cases cell-laden gels had one-half to one-seventh the maximum stress of their corresponding acellular gels. No cell-laden groups exceeded a maximum stress of 1500 $\mathrm{kPa}$, regardless of the mechanical performance of acellular gels with the same formulation. The shear modulus of cell-laden constructs is given in Figure 11. Unlike with maximum stress and strain percent, there were very few instances of cell-laden gels demonstrating decreased shear and compressive moduli in comparison to their corresponding acellular gels $(\mathrm{p}<0.05)$. The ratio of compressive modulus to shear modulus $(\mathrm{E} / \mathrm{G})$ is similarly reported in Figure 12, and the same general trends were observed between acellular and cellular gels as in shear and compressive moduli.

\section{Discussion}

High-strength hydrogels with the ability to encapsulate cells and provide a microenvironment conducive to ECM production are highly desirable for the development of cartilage regeneration methods. Although it is generally understood that synthetic hydrogels offer polymer network tunability with the adjustment of molecular weights and concentrations, it is important to understand the extent to which these adjustments influence mechanical and swelling properties for better formulation design. This study provided the degree to which three factors influenced properties of IPN hydrogels made of agarose and PEG-DA, demonstrating that most properties, including GAG content of encapsulated cells over a three-week period, are affected by the concentration of PEG-DA much more than the molecular weight of PEG-DA or concentration of agarose. It should be noted, however, that PEG-DA molecular weight was by far the most significant factor in determining the maximum stress and percent strain the gels could withstand before fracture. Additionally, this study demonstrated the possibility of increasing the mechanical strength of agarose-PEG IPNs without sacrificing cell viability or activity.

Our group previously showed that agarose-PEG-DA IPNs had shear and compressive moduli approximately four times higher than that of pure PEG-DA (molecular weight $=$ $2000 \mathrm{Da}$ ) with the same starting concentration and molecular weight $[15,16]$. In these previous studies, the pure PEG-DA samples were made from the same PEG-DA monomer solution in which the agarose gels were soaked, which reduced the PEG-DA solution concentration and yield from the nominal (initial) solution concentration, resulting in 
approximately equal PEG-DA solids content in the IPNs and PEG-DA gels. However, in this study, pure PEG-DA samples were prepared directly from the specified PEG-DA concentrations, which is why there was higher PEG-DA content (Fig. 5b) in the pure PEGDA gels than in the IPNs (due to thermodynamic exclusion [36, 37]) in this study and not in our previous studies. This is an important distinction because PEG-DA content was an important contributing factor to the compressive $(E)$ and especially shear $(G)$ moduli in the current study. For ease of comparison and reporting, the results in this study were organized by formulation name, reporting the initial PEG-DA monomer concentration rather than the final PEG-DA content of the gels. Therefore, there often appeared to be a decrease in IPN mechanical performance when compared to PEG-DA gels of the same initial PEG-DA monomer concentration and molecular weight. However, when mechanical properties were compared among groups of similar final PEG-DA content, there was always an improvement in $E$ and $G$ with IPNs over pure PEG-DA gels. For instance, it would be more appropriate to compare the IPN formulation $2 \% / 15 \%(2 \mathrm{k})$ to the pure PEG-DA formulation $0 \% / 10 \%(2 \mathrm{k})$ because they had a similar average PEG-DA solids content $(9.2 \%$ and $8.9 \%$, respectively), rather than compare the same IPN to the pure PEG-DA formulation 0\%/15\% (2k), which had a much higher PEG-DA solids content (13.3\%) and therefore better mechanical performance. In this particular case, the IPN $2 \% / 15 \%(2 \mathrm{k})$ had a shear modulus twice that of the PEG-DA gel $0 \% / 10 \%(2 \mathrm{k})(60.7 \mathrm{kPa}$ versus $28.1 \mathrm{kPa})$ and a compressive modulus four times higher $(198.3 \mathrm{kPa}$ versus $48.5 \mathrm{kPa})$. This increase was not as great as the increase that Gong and Osada[1] demonstrated with their double-network hydrogels of PAMPS-PAAm, or our double-network gels of methacrylated chondroitin sulfatepolyacrylamide [38]. However, the strength of such double-network hydrogels is believed to arise from energy dissipation through the fracturing of covalent bonds in the first network [39], which may limit their application for load-bearing tissue engineering applications such as cartilage regeneration. Nonetheless, the mechanical properties of agarose-PEG-DAIPNS were still greater than the additive sum of their components when comparing gels of similar final PEG-DA content, demonstrating synergistic gel strengthening as outlined in previous studies $[15,16]$. It was this synergistic network strengthening that led us to consider interactions of the three factors in the statistical analysis, however the impact of a factor while keeping the other factor(s) at any fixed level was largest for PEG-DA concentration for most properties.

Effective transport is an important parameter in hydrogels for cell encapsulation, and diffusion alone is generally regarded as the driving transport phenomenon in hydrogels not subject to forced flow conditions [40]. While mesh size affects solute diffusion, it cannot be directly observed in hydrogels using the same methods used for solids, such as scanning electron microscopy, because of the limitations of hydrogel sample preparation [41]. However, Waters and coworkers $[42,43]$ have used small-angle X-ray and neutron scattering (SAXS/SANS) to determine the network structure of PEG-based hydrogels. Their work concluded that PEG-DA hydrogels have a heterogeneous network containing rod-like crosslink junctions from which many PEG chains extend, analogous to a "molecular bottle brush" morphology. Because of this heterogeneity, the correlation of the permeability of PEG-DA hydrogels with a single length scale parameter is a gross idealization, and thus mesh sizes reported in this study should be interpreted in telling of their correlative value rather than an actual network dimension.

Mesh size was determined from mechanical and swelling properties, though the equations used in this study were originally developed for single-network hydrogels. Although the mesh sizes reported for IPN formulations were calculated from the swelling and mechanical data for the entire gel, only the physical properties of PEG-DA (characteristic ratio, backbone bond length, number of bonds between crosslinks, and polymer density) were used rather than averaged values of PEG-DA and agarose. The network structure of various 
concentrations of agarose gels has been studied extensively for the last 40 years, and values for agarose pore size have consistently been reported to be at least an order of magnitude greater than the mesh sizes of the pure PEG-DA gels used in this study [44, 45]. Therefore, if the two interlocked networks are completely independent of one another, the mesh size of the PEG-DA network may be the dominant limiting factor to cell nutrient/waste transport and diffusion of ECM molecules such as GAGs because it is so much smaller than agarose. This method thus provides a reasonable estimate of PEG-DA network mesh size as a correlative parameter in PEG-DA-agarose IPNs used for cell encapsulation.

Bryant and Anseth [14] investigated the impact of calculated mesh size on GAG distribution in poly(ethylene glycol) dimethacrylate (PEG-DM) hydrogels encapsulated with young bovine chondrocytes. While the reported mesh sizes in their study cannot be directly compared to those in the current study due to the possible differences of calculation methods and the lack of direct measurements, our results for mesh size and mass swelling ratio were very similar to those of Bryant and Anseth for our pure 10\% and 20\% PEG-DA gels (3400 Da molecular weight), and an increase in GAG content was also seen with an increase in calculated mesh size (or, alternatively, a decrease in crosslink density, see Equation 8). Increasing PEG-DA concentrations led to better mechanical performance, however it also resulted in smaller mesh sizes. Although PEG-DA concentration was the most influential factor for mesh size, there were many formulations that had a significantly higher mesh size $(\mathrm{p}<0.05)$ when the 6k PEG-DA molecular weight was used compared to lower molecular weights. An area of future investigation may be to use a PEG-DA molecular weight even higher than 6000 Da to determine whether it may increase the fracture performance of the hydrogels, and/or allow for using higher concentrations of PEG-DA (which may increase stiffness) while still possibly maintaining a reasonable crosslink density needed for proper GAG diffusion. However, the diffusion time would need to be reasonable, as it of course increases with molecular weight.

A preliminary study showed that the highest concentration of agarose possible for cell encapsulation was around $5 \% \mathrm{w} / \mathrm{v}$, as higher concentrations begin to gel at temperatures greater than $37{ }^{\circ} \mathrm{C}$. There was often an improvement $(\mathrm{p}<0.05)$ in $\mathrm{E}$ and $\mathrm{G}$ for IPNs containing 5\% agarose when compared to those containing 2\% agarose. However, in gels with the highest mechanical strength (those with a PEG-DA molecular weight of $6000 \mathrm{Da}$ ), mesh size and swelling were significantly lower in IPNs with 5\% agarose than in pure PEGDA gels or IPNs with $2 \%$ agarose $(p<0.05)$. Furthermore, the high viscosity of $5 \%$ agarose prior to gelation makes it difficult to work with during the encapsulation process. The increase in stiffness given by IPNs with 5\% agarose is likely not worth the trade-off of the increased cost of supplies, difficulty of use, and decreased GAG distribution due to a smaller mesh size [14], especially since the increase in agarose concentration does not significantly improve the maximum stress.

Constructs containing cells did not differ significantly $(p<0.05)$ in moduli from acellular gels in most cases and generally followed the same trends with respect to network concentrations and PEG-DA molecular weights. Furthermore, cell-laden gels did not have significantly different shear or compressive moduli between the week 0 and 3 time points (with the exception of one formulation in shear modulus), suggesting that decreases in mechanical properties were not caused by long-term cell metabolism. This was true even for the $2 \% /$ $10 \%$ (6k) group, the formulation that showed a 12-fold increase in normalized GAG deposition from 0 to 3 weeks. Ideally, cell-laden constructs would either begin with a stiffness similar to native cartilage or approach it as ECM is produced. Although the gels did not decrease in stiffness with the incorporation of live cells, the question remains as to whether or not stiffness can eventually increase if given time for sufficient ECM production in IPNs. Han and coworkers[46] recently reported that the incorporation of sulfated GAG 
and type II collagen into $2 \%$ agarose constructs significantly improved their stiffness and peak stress, especially when used in combination. However, the concentration of GAG used was $2.5 \mathrm{mg} / \mathrm{mL}$, which was much higher than the concentration of cell-produced GAG $(0.11$ $\mathrm{mg} / \mathrm{mL}$ for the highest-performing group) in week 3 of the current study. The potential for increasing the stiffness of cell-laden agarose-PEG-DA IPNs, perhaps by manipulating cell performance, e.g., via the incorporation of methacrylated chondroitin sulfate [15] or aggrecan [47], may be worth further investigation. Therefore, it is our expectation that both mechanical and cell performance can be tuned semi-independently by the altering IPN composition and bioactive moieties, respectively, while acknowledging that cell performance over time is expected to strongly influence the long-term mechanical performance.

The E/G ratio and degree of linearity on the stress versus strain function plot are good measures of whether a hydrogel may be classified as an ideal elastomer. Hydrogels with an E/G ratio of three are considered to be ideal elastomers, though they are typically slightly above three in compression testing [35]. The trends in E/G ratios (Fig. 3b) were caused by a systematic deviation in linearity of the stress-strain function profiles (Fig. 4), however deviations did not typically begin until a strain function of $\sim 4$, corresponding to $\sim 55 \%$ strain, which still demonstrates quite ideal elastic behavior as stated in our previous work [15, 16]. The shear modulus (G, Fig. 3a) was analyzed as the slope of the stress versus strain function $\left(\lambda-1 / \lambda^{2}\right)$ profiles up to the same strain function value of $10(69 \%$ strain) for all 27 formulations to determine if, in addition to swelling and compressive modulus, there were any systematic trends in the stress-strain function profiles. A strain function value of 10 was used as the upper limit of the range, as pure PEG-DA gels showed a high degree of linearity up to this value. This analysis revealed that the trends in E/G ratios (Figures $3 \mathrm{~b}$ and 4 ) increased with increasing concentrations of agarose as well as increasing PEGDA molecular weight. The reported elevations in $\mathrm{E} / \mathrm{G}$ ratios led to an underestimation in the shear modulus ( $\mathrm{G}$, by an average of $10 \%)$, which in turn led to an underestimation in crosslink density ( $\rho_{\mathrm{x}}$, Fig. 6 b, by an average of $13 \%$ ) and a concomitant overestimation in the polymer-solvent interaction parameter ( $\mathrm{X}$, by less than $1 \%$ on average), though the trends and overall conclusions of these G-dependent parameters are essentially unaffected. It is possible that another model for determining G, such as the Mooney-Rivlin model [48], could better fit the stress-strain function profiles of agarose/PEG-DA IPNs. This will be the subject of further analysis, focused on the modulus alone, rather than the larger scope of this paper.

The polymer-solvent interaction parameter $(\mathrm{X})$ is typically used as an intermediate for calculating the molecular weight between crosslinks. Many studies on PEG derivatives [14, $17,22,23,25,28,32,33$ ] have used a value of 0.426 , reported by Merrill and coworkers [49] in 1993, though more recent studies have reported values of $\sim 0.47$ under similar conditions [50, 51]. Our calculations of all 27 formulations averaged to a value of $0.510 \pm$ 0.002 , which is in agreement with our previous work, though higher than the value typically used. As with the $\mathrm{E} / \mathrm{G}$ ratio, the calculation of $X$ was used for confirming the elastic ideality of our IPNs, and the systematic increases of E/G matched those of X. Because there were small overestimations of $X$ in formulations with higher $E / G$ ratios, the average value of $X$ for all 27 formulations is reasonably higher than what has been previously used for singlenetwork hydrogels.

Of concern is the observation that hydrogels with the greatest fracture properties had a significant decrease in maximum stress when encapsulated with cells. Regardless of how the corresponding acellular gels performed, gels with encapsulated cells did not exceed a maximum stress of $1500 \mathrm{kPa}$, suggesting a possible limit intrinsic to agarose/PEG-DA IPNs for load-bearing applications such as cartilage tissue engineering. As previously mentioned, stress-strain curves for these formulations increased sharply beyond a strain of 50\%, 
resulting in large differences in maximum stress between cellular and acellular gels for seemingly-small differences in maximum strain percent. Gels with the highest maximum stress withstood over $80 \%$ strain in compression, so even slight defects could lead to dramatically premature failure. For this reason, we recently reviewed fracture mechanics studies of both cartilage and hydrogels and recommended alternate methods of determining apparent fracture toughness such as a Mode I modified single edge notch test [52]. The presence of chondrocytes may have reduced polymerization efficiency during photopolymerization, not only because they added opacity to the gels, reducing UV intensity but also because their size is much larger than typical distances between crosslinks in PEGDA hydrogels. Cho and coworkers [53] also concluded that encapsulated materials alter PEG network formation as a result of the heterogeneity of the hydrogels, leading to microfractures/defects that resulted in a functional pore size as high as 20 times their calculated theoretical mesh size. The group later went on to investigate the beneficial increased network permeability caused by the defects and determined that fewer network defects were present in PEG gels with a higher solids content (30-40\%) [54]. The presence of cells decreasing the maximum stress demonstrates the importance of not only determining fracture properties of cell-laden hydrogels for tissue engineering, but also of investigating particle size and concentration when considering the addition supplemental materials to hydrogels for purposes such as increasing cell viability or ECM production.

The exploration of higher agarose and PEG-DA concentrations as well as higher PEG-DA molecular weights have demonstrated vastly improved (an order of magnitude, in some instances) mechanical properties of agarose/PEG-DA IPNs and pure PEG-DA gels over our previous studies, while maintaining cell viability and improving cell activity. By reporting the extent to which statistically significant factors improved mechanical properties, swelling characteristics, and network parameters, as well as displaying where the significant differences among formulations are prevalent for each factor, we have gained a better understanding of how to address the limitations of competing properties for IPN design. Studies have recently reported methods for synthesizing PEG $[14,55,56]$ and agarose [57, 58] networks with controllable biodegradation. These studies suggest that cell-laden agarose-PEG-DA IPNs can likely be designed for in vivo degradation in parallel with ECM production. A vast variety of natural biomaterials have also been studied for tissue engineering applications [59], and additional studies that incorporate 'raw materials' into agarose-PEG-DA IPNs may further enhance their already-promising properties.

\section{Conclusions}

This study demonstrated the possibility of mechanical strength enhancement in agarosePEG-DA IPNs by varying the parameters of agarose concentration, PEG-DA concentration, and PEG-DA molecular weight. It was determined that the most significant factor in determining IPN compressive and shear moduli was PEG-DA concentration, while the most significant factor in determining IPN failure properties was the molecular weight of the PEG-DA. It was also noteworthy that the IPN composition had little effect on cell performance, with the exception of increased GAG synthesis with larger mesh sizes. Although acellular IPNs had superior mechanical integrity compared to pure PEG-DA gels of similar final PEG-DA content, the maximum stress of cell-laden hydrogels did not exceed 1.5 MPa, regardless of the performance of corresponding acellular formulations. This finding demonstrates the need to explore methods to overcome this barrier, perhaps by incorporating bioactive 'raw materials' for increased GAG production and maximum stress. In the future, it is our expectation that both mechanical and cell performance can be tuned semi-independently by the altering IPN composition and bioactive moieties, respectively, which together with complementary strategies such as incorporating degradable sequences may provide a new platform technology for cartilage regeneration. 


\section{Acknowledgments}

We gratefully acknowledge funding from the NIH directly (R21 EB008783, R01 AR056347) and via the KU IMSD program (R25 GM062232). This material is also based on work supported by the National Science Foundation under Grant No. IOS 0805264. Any opinions, findings, and conclusions or recommendations expressed in this article are those of the authors and do not necessarily reflect the views of the National Science Foundation. We also acknowledge funding from the Self Graduate Research Fellowship for the funding of A.N.R.

\section{References}

1. Gong JP, Katsuyama Y, Kurokawa T, Osada Y. Double-network hydrogels with extremely high mechanical strength. Adv Mater. 2003; 15:1155-8.

2. Na Y-H, Kurokawa T, Katsuyama Y, Tsukeshiba H, Gong JP, Osada Y, et al. Structural characteristics of double network gels with extremely high mechanical strength. Macromolecules. 2004; 37:5370-4.

3. Myung D, Waters D, Wiseman M, Duhamel P-E, Noolandi J, Ta CN, et al. Progress in the development of interpenetrating polymer network hydrogels. Polym Adv Technol. 2008; 19:64757. [PubMed: 19763189]

4. Gong JP. Why are double network hydrogels so tough? Soft Matter. 2010; 6:2583-90.

5. Azuma C, Yasuda K, Tanabe Y, Taniguro H, Kanaya F, Nakayama A, et al. Biodegradation of hightoughness double network hydrogels as potential materials for artificial cartilage. J Biomed Mater Res A. 2007; 81A:373-80. [PubMed: 17117467]

6. Tanabe Y, Yasuda K, Azuma C, Taniguro H, Onodera S, Suzuki A, et al. Biological responses of novel high-toughness double network hydrogels in muscle and the subcutaneous tissues. J Mater Sci Mater Med. 2008; 19:1379-87. [PubMed: 17914620]

7. Ronken S, Wirz D, Daniels A, Kurokawa T, Gong J, Arnold M. Double-network acrylamide hydrogel compositions adapted to achieve cartilage-like dynamic stiffness. Biomech Model Mechanobiol. :1-6.

8. Myung D, Koh W, Ko J, Hu Y, Carrasco M, Noolandi J, et al. Biomimetic strain hardening in interpenetrating polymer network hydrogels. Polymer. 2007; 48:5376-87.

9. Yasuda K, Kitamura N, Gong JP, Arakaki K, Kwon HJ, Onodera S, et al. A novel double-network hydrogel induces spontaneous articular cartilage regeneration in vivo in a large osteochondral defect. Macromol Biosci. 2009; 9:307-16. [PubMed: 19031389]

10. Wang Q, Hou RX, Cheng YJ, Fu J. Super-tough double-network hydrogels reinforced by covalently compositing with silica-nanoparticles. Soft Matter. 2012; 8:6048-56.

11. Zhang X, Guo X, Yang S, Tan S, Li X, Dai H, et al. Double-network hydrogel with high mechanical strength prepared from two biocompatible polymers. J Appl Polym Sci. 2009; 112:3063-70.

12. Tang Y, Sun J, Fan H, Zhang X. An improved complex gel of modified gellan gum and carboxymethyl chitosan for chondrocytes encapsulation. Carbohydr Polym. 2012; 88:46-53.

13. Shin H, Olsen BD, Khademhosseini A. The mechanical properties and cytotoxicity of cell-laden double-network hydrogels based on photocrosslinkable gelatin and gellan gum biomacromolecules. Biomaterials. 2012; 33:3143-52. [PubMed: 22265786]

14. Bryant SJ, Anseth KS. Hydrogel properties influence ECM production by chondrocytes photoencapsulated in poly(ethylene glycol) hydrogels. J Biomed Mater Res. 2002; 59:63-72. [PubMed: 11745538]

15. Ingavle G, Dormer N, Gehrke S, Detamore M. Using chondroitin sulfate to improve the viability and biosynthesis of chondrocytes encapsulated in interpenetrating network (IPN) hydrogels of agarose and poly(ethylene glycol) diacrylate. J Mater Sci Mater Med. 2012; 23:157-70. [PubMed: 22116661]

16. DeKosky BJ, Dormer NH, Ingavle GC, Roatch CH, Lomakin J, Detamore MS, et al. Hierarchically designed agarose and poly(ethylene glycol) interpenetrating network hydrogels for cartilage tissue engineering. Tissue Eng Part C Methods. 2010; 16:1533-42. [PubMed: 20626274] 
17. Nguyen QT, Hwang Y, Chen AC, Varghese S, Sah RL. Cartilage-like mechanical properties of poly (ethylene glycol)-diacrylate hydrogels. Biomaterials. 2012; 33:6682-90. [PubMed: 22749448]

18. Weng L, Liang S, Zhang L, Zhang X, Xu J. Transport of glucose and poly(ethylene glycol)s in agarose gels studied by the refractive index method. Macromolecules. 2005; 38:5236-42.

19. Wang L, Lazebnik M, Detamore MS. Hyaline cartilage cells outperform mandibular condylar cartilage cells in a TMJ fibrocartilage tissue engineering application. Osteoarthr Cartil. 2009; 17:346-53. [PubMed: 18760638]

20. Wang L, Detamore MS. Effects of growth factors and glucosamine on porcine mandibular condylar cartilage cells and hyaline cartilage cells for tissue engineering applications. Arch Oral Biol. 2009; 54:1-5. [PubMed: 18640663]

21. Gehrke, SH. Synthesis and properties of hydrogels used for drug delivery. In: GL, Amidon; PI, Lee; EM, Topp, editors. Transport Processes in Pharmaceutical Systems. New York: Marcel Dekker; 2000. p. 473-546.

22. Jeerage KM, LaNasa SM, Hughes HA, Lauria DS, Bryant SJ, Slifka AJ. Scanning electrochemical microscopy measurements of photopolymerized poly(ethylene glycol) hydrogels. Polymer. 2010; 51:5456-61.

23. Avens H, Chang E, May A, Berron B, Seedorf G, Balasubramaniam V, et al. Fluorescent polymeric nanocomposite films generated by surface-mediated photoinitiation of polymerization. $\mathrm{J}$ Nanopart Res. 2011; 13:331-46.

24. Behravesh E, Sikavitsas VI, Mikos AG. Quantification of ligand surface concentration of bulkmodified biomimetic hydrogels. Biomaterials. 2003; 24:4365-74. [PubMed: 12922149]

25. Mellott MB, Searcy K, Pishko MV. Release of protein from highly cross-linked hydrogels of poly(ethylene glycol) diacrylate fabricated by UV polymerization. Biomaterials. 2001; 22:929-41. [PubMed: 11311012]

26. Villanueva I, Klement BJ, von Deutsch D, Bryant SJ. Cross-linking density alters early metabolic activities in chondrocytes encapsulated in poly(ethylene glycol) hydrogels and cultured in the rotating wall vessel. Biotechnol Bioeng. 2009; 102:1242-50. [PubMed: 18949761]

27. Temenoff JS, Athanasiou KA, Lebaron RG, Mikos AG. Effect of poly(ethylene glycol) molecular weight on tensile and swelling properties of oligo(poly(ethylene glycol) fumarate) hydrogels for cartilage tissue engineering. J Biomed Mater Res. 2002; 59:429-37. [PubMed: 11774300]

28. Lu S, Anseth KS. Release behavior of high molecular weight solutes from poly(ethylene glycol)based degradable networks. Macromolecules. 2000; 33:2509-15.

29. Rubenstein, M.; Colby, R. Polymer physics. Oxford Univ. Press; New York: 2003.

30. Sperling, LH. Introduction to Physical Polymer Science. 4th ed.. Hoboken, NJ: John Wiley \& Sons, Inc; 2006.

31. Peppas, NA.; Barr-Howell, BD. Characterization of the crosslinked structure of hydrogels. In: NA, Peppas, editor. Hydrogels in Medicine and Pharmacy. Boca Raton, FL: CRC Press; 1986. p. 27-56.

32. Russell RJ, Axel AC, Shields KL, Pishko MV. Mass transfer in rapidly photopolymerized poly(ethylene glycol) hydrogels used for chemical sensing. Polymer. 2001; 42:4893-901.

33. Hickey AS, Peppas NA. Mesh size and diffusive characteristics of semicrystalline poly(vinyl alcohol) membranes prepared by freezing/thawing techniques. J Memb Sci. 1995; 107:229-37.

34. Betancourt T, Pardo J, Soo K, Peppas NA. Characterization of $\mathrm{pH}$-responsive hydrogels of poly(itaconic acid-g-ethylene glycol) prepared by UV-initiated free radical polymerization as biomaterials for oral delivery of bioactive agents. J Biomed Mater Res A. 2010; 93A:175-88. [PubMed: 19536838]

35. Xue W, Huglin MB, Jones TGJ. Swelling and network parameters of crosslinked thermoreversible hydrogels of poly(N-ethylacrylamide). Eur Polym J. 2005; 41:239-48.

36. Albertsson, PA. Partition of cell particles and macromolecules. 2nd ed. New York: WileyInterscience; 1986.

37. Gehrke SH, Vaid NR, McBride JF. Protein sorption and recovery by hydrogels using principles of aqueous two-phase extraction. Biotechnol Bioeng. 1998; 58:416-27. [PubMed: 10099276] 
38. Suekama TC, Hu J, Kurokawa T, Gong JP, Gehrke SH. Double-network strategy improves fracture properties of chondroitin sulfate networks. ACS Macro Lett. 2013; 2:137-40.

39. Brown HR. A model of the fracture of double network gels. Macromolecules. 2007; 40:3815-8.

40. Slaughter BV, Khurshid SS, Fisher OZ, Khademhosseini A, Peppas NA. Hydrogels in regenerative medicine. Adv Mater. 2009; 21:3307-29. [PubMed: 20882499]

41. Green RA, Baek S, Poole-Warren LA, Martens PJ. Conducting polymer-hydrogels for medical electrode applications. Sci Technol Adv Mater. 2010; 11:014107.

42. Waters DJ, Engberg K, Parke-Houben R, Hartmann L, Ta CN, Toney MF, et al. Morphology of photopolymerized end-linked polyethylene glycol) hydrogels by small-angle X-ray scattering. Macromolecules. 2010; 43:6861-70. [PubMed: 21403767]

43. Waters DJ, Engberg K, Parke-Houben R, Ta CN, Jackson AJ, Toney MF, et al. Structure and mechanism of strength enhancement in interpenetrating polymer network hydrogels. Macromolecules. 2011; 44:5776-87.

44. Jokerst JV, Chou J, Camp JP, Wong J, Lennart A, Pollard AA, et al. Location of biomarkers and reagents within agarose beads of a programmable nio-nano-chip. Small. 2011; 7:613-24. [PubMed: 21290601]

45. Pluen A, Netti PA, Jain RK, Berk DA. Diffusion of macromolecules in agarose gels: comparison of linear and globular gonfigurations. Biophys J. 1999; 77:542-52. [PubMed: 10388779]

46. Han E, Ge C, Chen AC, Schumacher BL, Sah RL. Compaction enhances extracellular matrix content and mechanical properties of tissue-engineered cartilaginous constructs. Tissue Eng Part A. 2012; 18:1151-60. [PubMed: 22372815]

47. Ingavle GC, Frei A, Gehrke SH, Detamore MS. Incorporation of aggrecan in interpenetrating network hydrogels to improve cellular performance for cartilage tissue engineering. Tissue Eng Part A. 2013

48. Mooney M. A theory of large elastic deformation. J Appl Phys. 1940:11.

49. Merrill EW, Dennison KA, Sung C. Partitioning and diffusion of solutes in hydrogels of polyethylene oxide). Biomaterials. 1993; 14:1117-26. [PubMed: 8130315]

50. Baulin VA, Halperin A. Concentration dependence of the Flory $X$ parameter within two-state models. Macromolecules. 2002; 35:6432-8.

51. Eliassi A, Modarress H, Mansoori GA. Measurement of activity of water in aqueous polyethylene glycol) solutions (effect of excess volume on the Flory-Huggins X-parameter). J Chem Eng Data. 1998; 44:52-5.

52. Xiao Y, Friis LA, Gehrke SH, Detamore MS. Mechanical testing of hydrogels in cartilage tissue engineering: Beyond the compressive modulus. Tissue Eng Part B Rev. 2013

53. Nam-Joon C, Menashe E, Anming X, Wonjae L, Eric C, Julie B, et al. Viral infection of human progenitor and liver-derived cells encapsulated in three-dimensional PEG-based hydrogel. Biomed Mater. 2009; 4:011001. [PubMed: 18981544]

54. Lee W, Cho N-J, Xiong A, Glenn JS, Frank CW. Hydrophobic nanoparticles improve permeability of cell-encapsulating polyethylene glycol) hydrogels while maintaining patternability. Proc Natl Acad Sci U S A. 2010; 107:20709-14. [PubMed: 21071674]

55. Roberts J, Nicodemus G, Greenwald E, Bryant S. Degradation improves tissue formation in (unloaded chondrocyte-laden hydrogels. Clin Orthop Relat Res. 2011; 469:2725-34. [PubMed: 21347817]

56. Bryant SJ, Bender RJ, Durand KL, Anseth KS. Encapsulating chondrocytes in degrading PEG hydrogels with high modulus: Engineering gel structural changes to facilitate cartilaginous tissue production. Biotechnol Bioeng. 2004; 86:747-55. [PubMed: 15162450]

57. Zhang L-M, Wu C-X, Huang J-Y, Peng X-H, Chen P, Tang S-Q. Synthesis and characterization of a degradable composite agarose/HA hydrogel. Carbohydr Polym. 2012; 88:1445-52.

58. De Rosa E, Urciuolo F, Borselli C, Gerbasio D, Imparato G, Netti PA. Time and space evolution of transport properties in agarose-chondrocyte constructs. Tissue Eng. 2006; 12:2193-201. [PubMed: 16968160]

59. Renth AN, Detamore MS. Leveraging "raw materials" as building blocks and bioactive signals in regenerative medicine. Tissue Eng Part B Rev. 2012; 18:341-62. [PubMed: 22462759] 
a

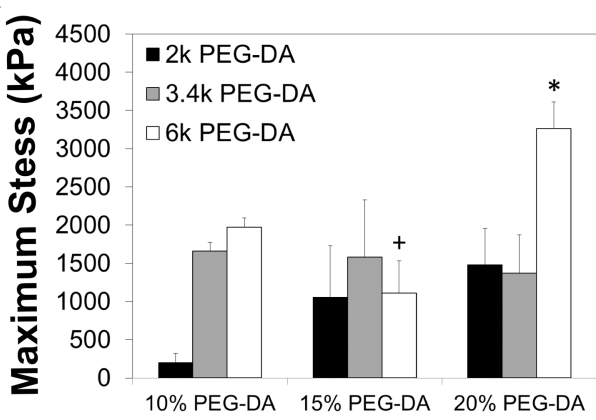

$0 \%$ Agarose

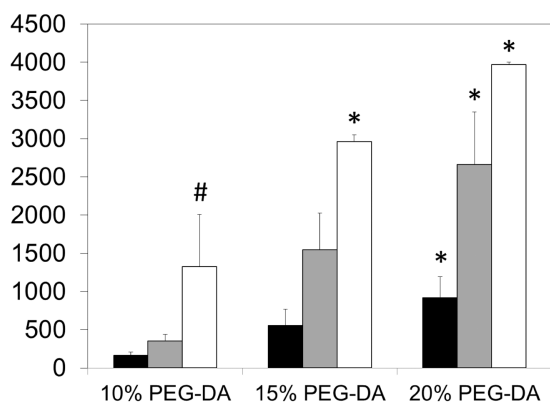

$5 \%$ Agarose

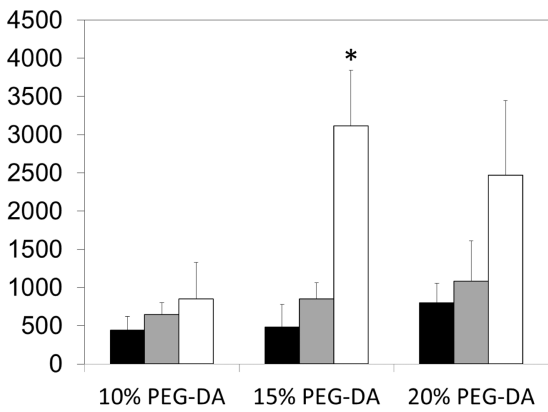

b
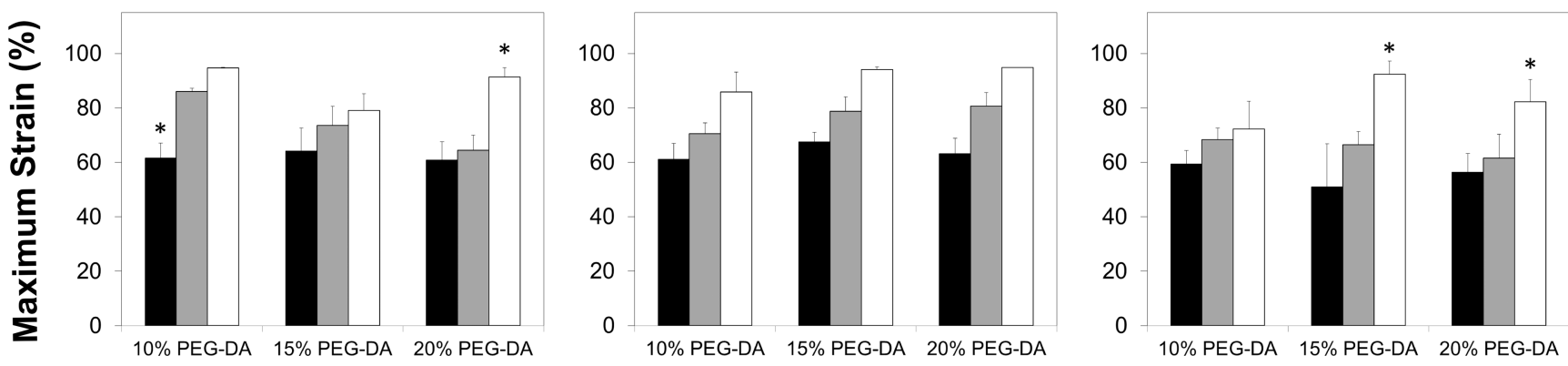

Figure 1.

(a) Maximum stress and (b) maximum strain percent of agarose and PEG-DA IPNs with PEG-DA molecular weights of 2000 (2k), 3400 (3.4k), and 6000 (6k) Da and agarose concentrations of $0 \%$ (pure PEG-DA gels), 2\%, and 5\%. Unlike most mechanical and swelling characteristics, PEG-DA molecular weight was the most significantly-contributing factor for these two failure properties. Values represent mean \pm standard deviation with $n=4$. Values significantly different $(\mathrm{p}<0.05)$ from groups varying only in: "PEG-DA concentration, ${ }^{+}$agarose concentration, *PEG-DA molecular weight. 


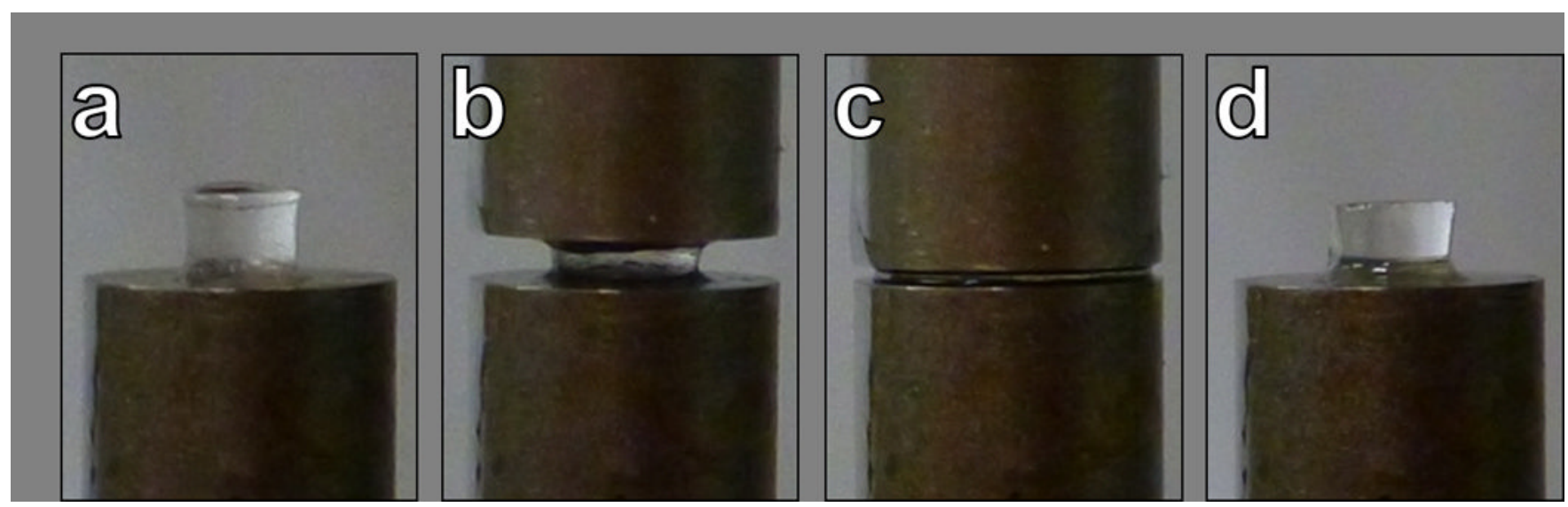

Figure 2.

Images of an IPN sample (2\% agarose, 20\% 6k PEG-DA) (a) before unconfined compression, (b) at 50\% strain, (c) at 95\% strain, and (d) immediately after compression. This particular sample had a compressive modulus of $313 \mathrm{kPa}$ and withstood a stress of 4.2 MPa without fracturing. After soaking in a phosphate buffered saline solution for 1 hour, the sample recovered to approximately $97 \%$ of its original height. 
a

2k PEG-DA

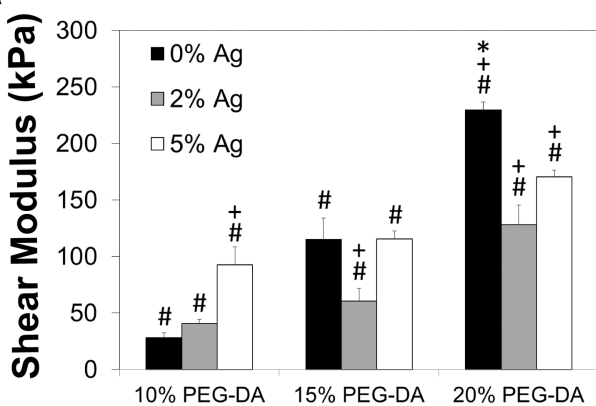

3.4k PEG-DA

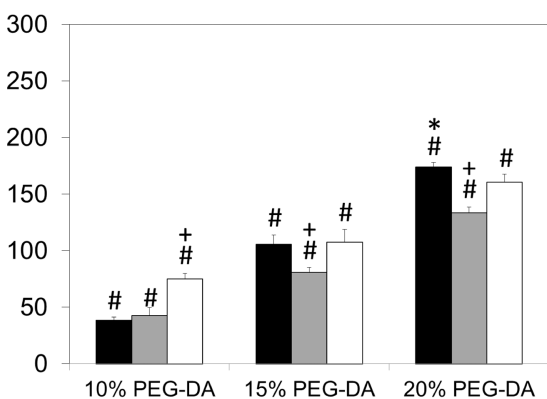

6k PEG-DA

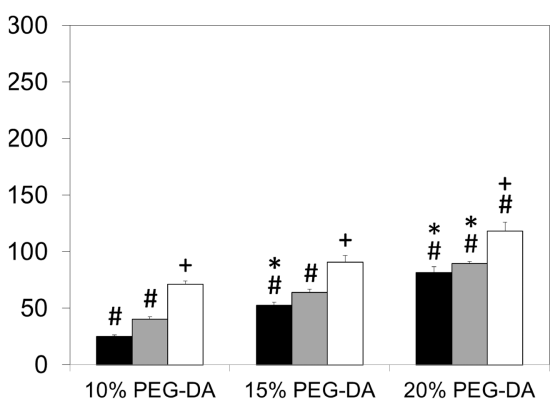

b
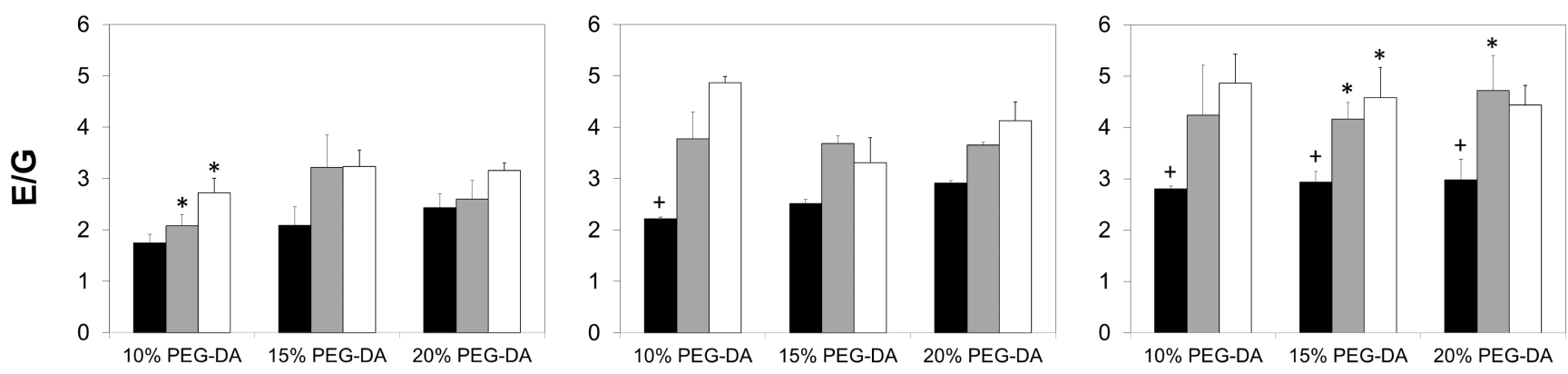

Figure 3.

(a) Shear modulus and (b) ratio of compressive modulus to shear modulus of agarose (Ag) and PEG-DA IPNs. Nearly every formulation had a significantly higher shear modulus $(\mathrm{p}<0.05)$ with an increase in PEG-DA concentration, while E/G generally increased with increases in agarose concentration and PEG-DA molecular weight. Values represent mean \pm standard deviation with $n=4$. Values significantly different $(p<0.05)$ among groups varying only in: ${ }^{\text {PEG-DA concentration, }}{ }^{+}$agarose concentration, *PEG-DA molecular weight. 

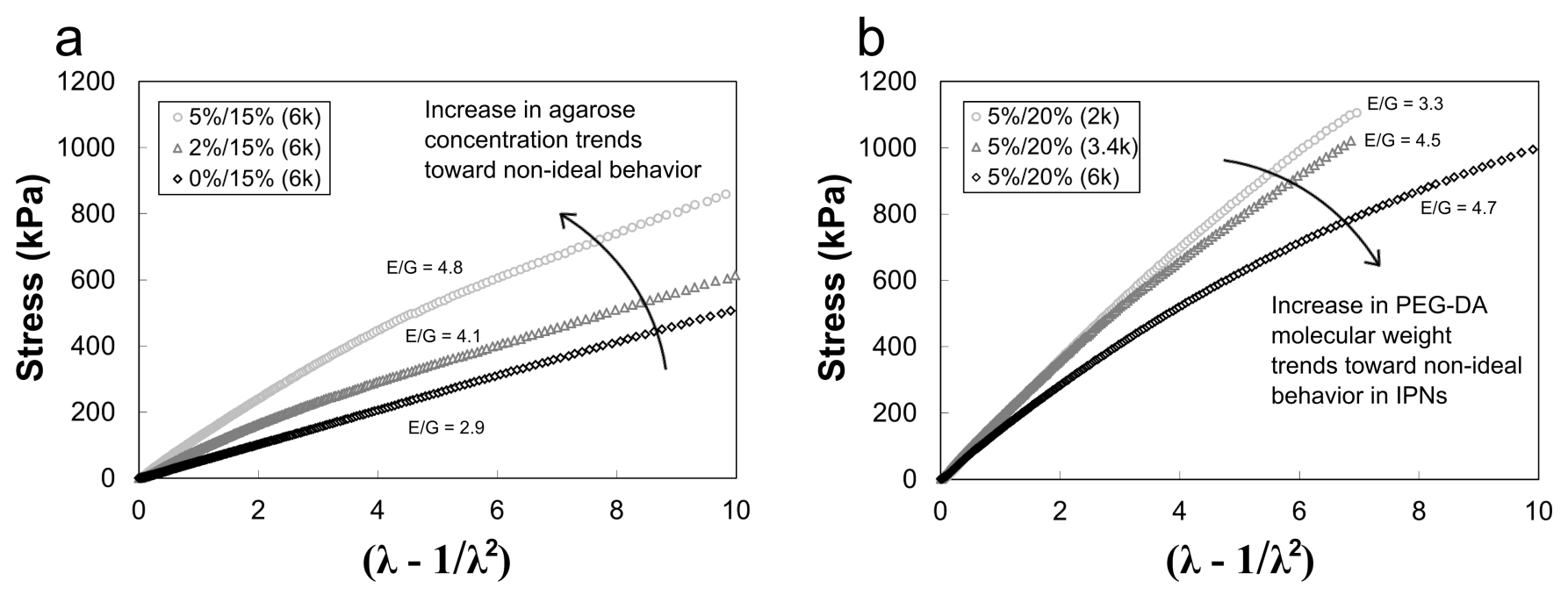

Figure 4.

Representative neo-Hookean elasticity model plots of stress versus strain function, demonstrating trends toward non-ideal elastic behavior for (a) increases in agarose concentration and (b) increases in PEG-DA molecular weight (in IPNs). The shear modulus, $\mathrm{G}$, was taken as the slope of these plots up to a strain function value of 10 or until fracture. An $\mathrm{E} / \mathrm{G}$ ratio of 3 is considered ideal elastic behavior, as is a linear stress-strain function profile. PEG-DA = polyethylene glycol) diacrylate, $\lambda=\mathrm{L}_{\mathrm{L}}$, IPN = interpenetrating network, data points after fracture omitted in panel $b$. 
a

b 3.4k PEG-DA

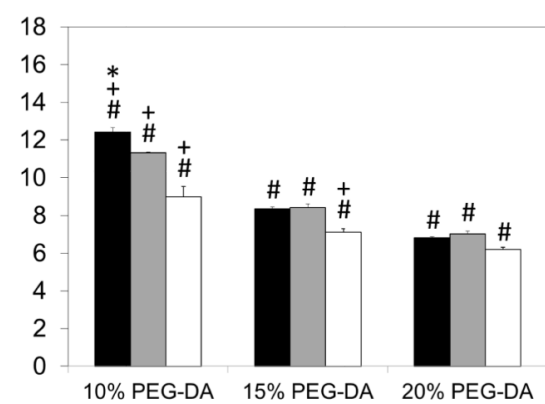

6k PEG-DA

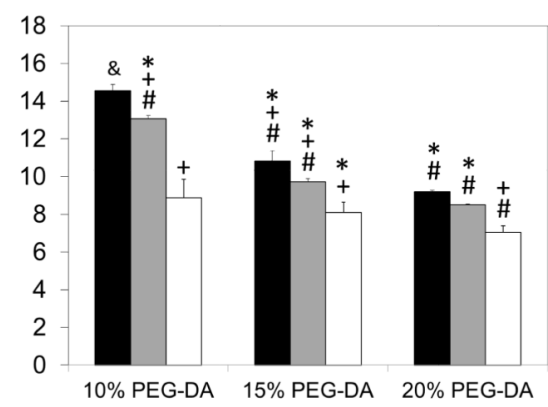

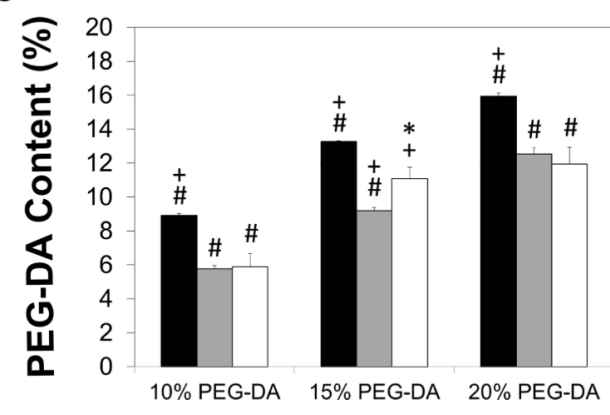
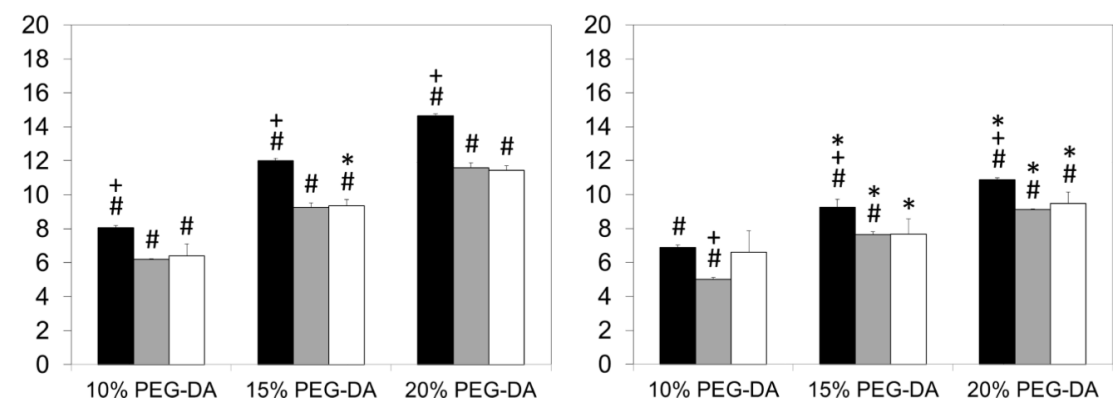

Figure 5.

(a) Equilibrium mass swelling ratio and (b) PEG-DA solids content. The mass swelling ratios generally showed an inverse relationship with shear modulus (Figure 2a), and the decreased PEG-DA content in IPNs versus pure PEG-DA gels (0\% Ag) helps to explain corresponding decreases in shear modulus. Values represent mean \pm standard deviation with $\mathrm{n}=4$. Values significantly different $(\mathrm{p}<0.05)$ from groups varying only in: \#PEG-DA concentration, ${ }^{+}$agarose concentration, *PEG-DA molecular weight; ${ }^{\&}$ value significantly higher $(\mathrm{p}<0.05)$ than all 26 other formulations. 
a

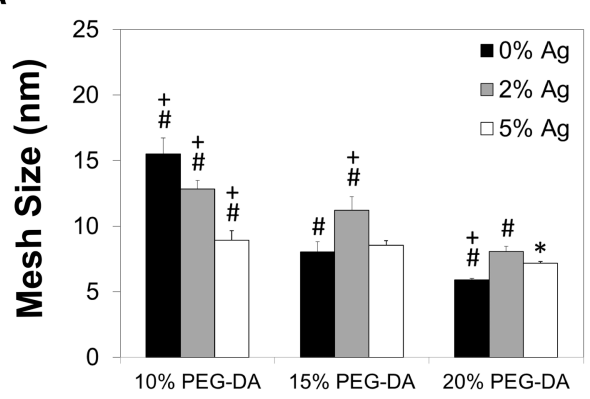

2k PEG-DA

b

Figure 6. formulations. 3.4k PEG-DA

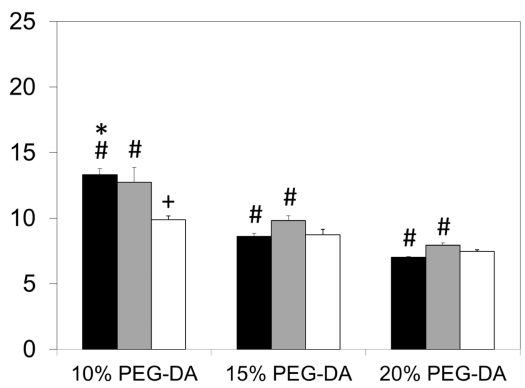

6k PEG-DA

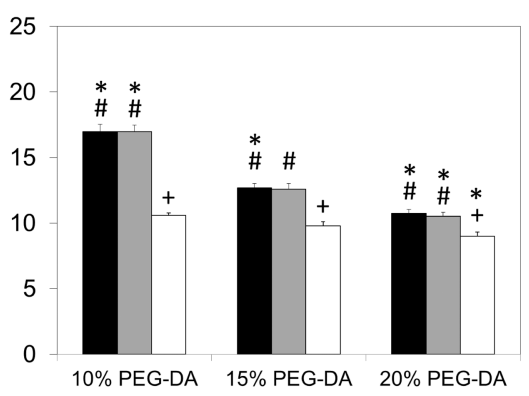

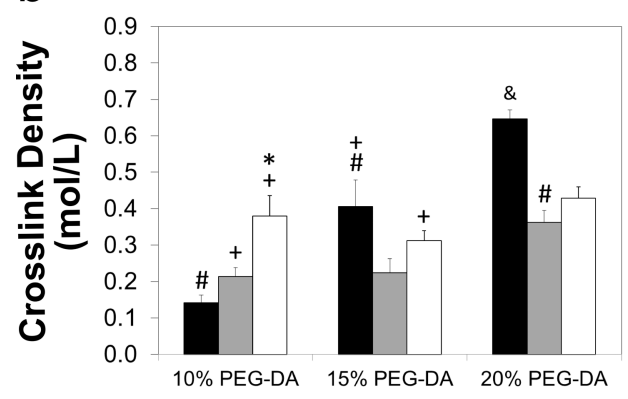
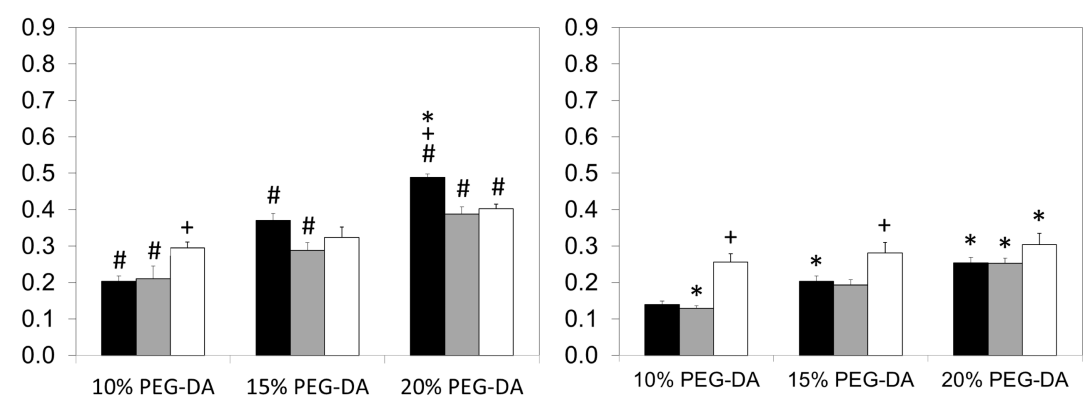

(a) Mesh size and (b) crosslink density of PEG-DA in IPNs. Both properties were most significantly influenced by PEG-DA, though molecular weight was also an important factor. Values represent mean \pm standard deviation with $n=4$. Values significantly different $(p<0.05)$ from groups varying only in: "PEG-DA concentration, ${ }^{+}$agarose concentration, *PEG-DA molecular weight; ${ }^{\&}$ value significantly higher $(p<0.05)$ than all 26 other 

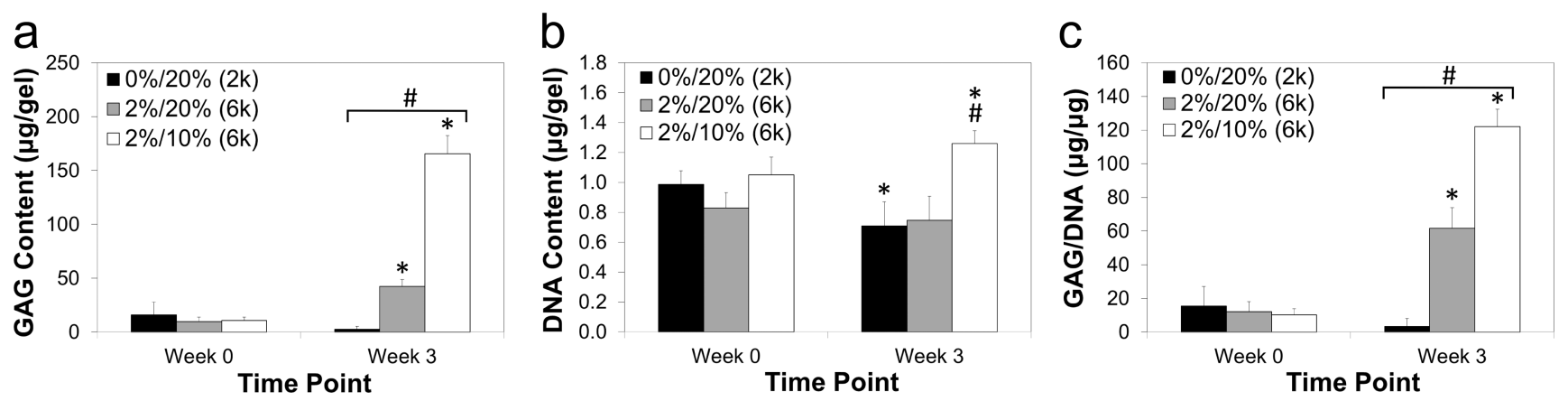

Figure 7.

(a) total GAG accumulated per gel, (b) DNA content per gel, and (c) GAG normalized to DNA content per gel encapsulated with chondrocytes at 0 and 3 weeks, with formulations listed as agarose concentration/PEG-DA concentration (PEG-DA molecular weight).

Halving the PEG-DA concentration in the $6 \mathrm{k}$ MW gels resulted in approximately double the normalized GAG accumulation for the two IPNs, while the pure PEG-DA gel did not provide a productive environment for chondrocytes in the absence of agarose. Values represent mean \pm standard deviation with $n=4$. *Values significantly different from week 0 time point $(p<0.005)$, \#significant differences $(p<0.05)$ among groups at the same time point. GAG, glycosaminoglycan. 

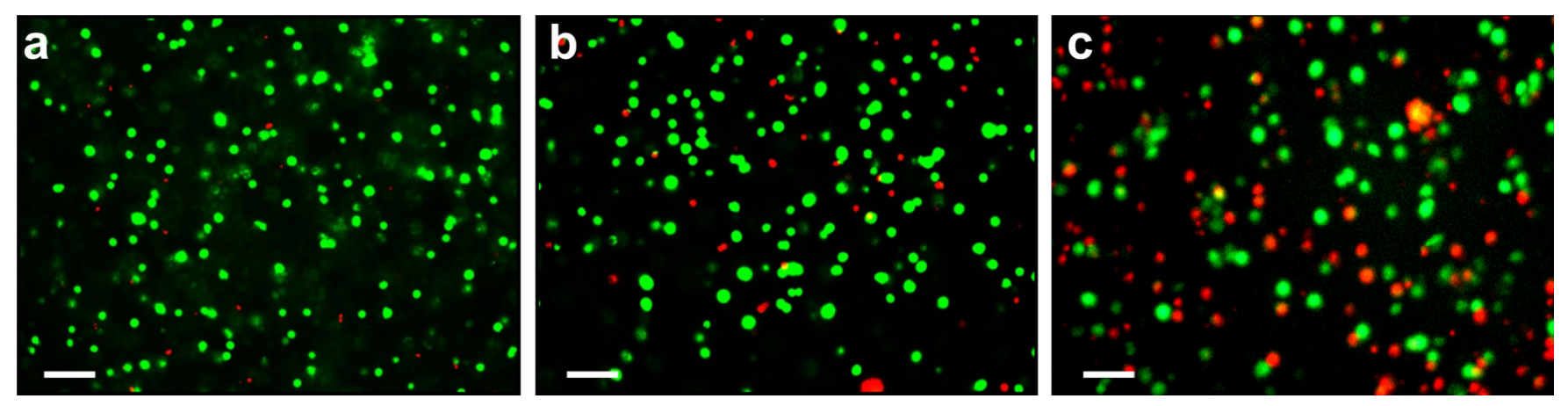

Figure 8.

Representative live/dead images for (a) agarose and (b) an IPN with 2\% agarose and 20\% PEG-DA (6000 Da molecular weight) 24 hours after encapsulation, and (c) an IPN with the same formulation 3 weeks after encapsulation. High cell viability 24 hours after encapsulation, even in the IPN formulation with the longest PEG-DA diffusion time and highest PEG-DA concentration, demonstrates the potential for this encapsulation process to work well with a wide variety of PEG-DA concentrations and molecular weights. Live cells are stained green with Calcein AM (green), while dead cells are stained red with ethidium homodimer-1. Scale bars $=50 \mu \mathrm{m}$. 
a

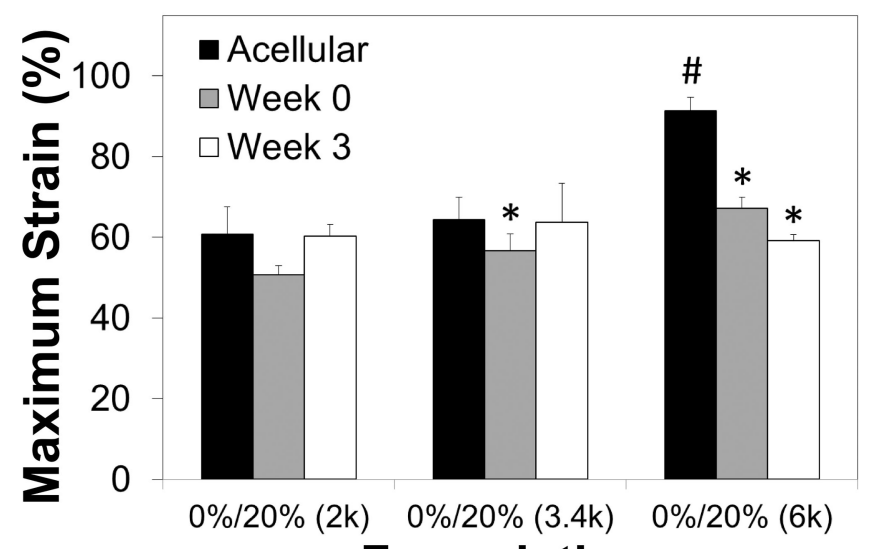

Formulation

Figure 9. the same panel $(\mathrm{p}<0.05)$. $\mathrm{b}$

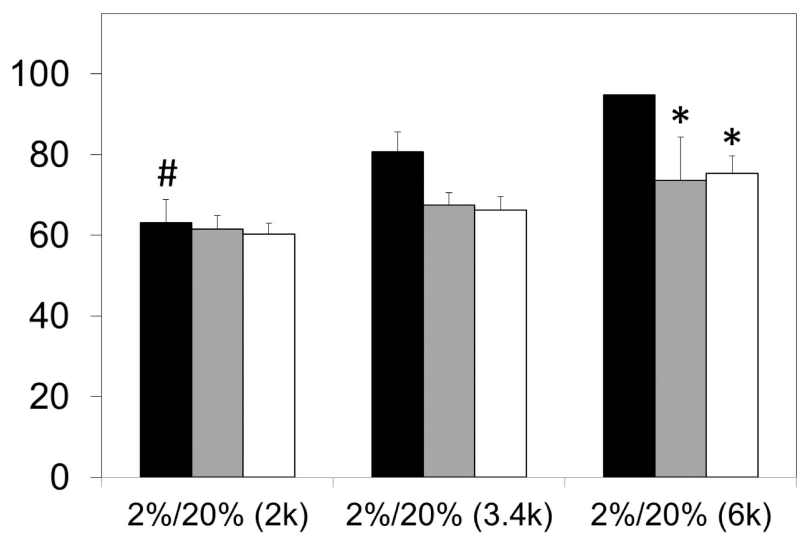

Formulation$$
\text { d }
$$

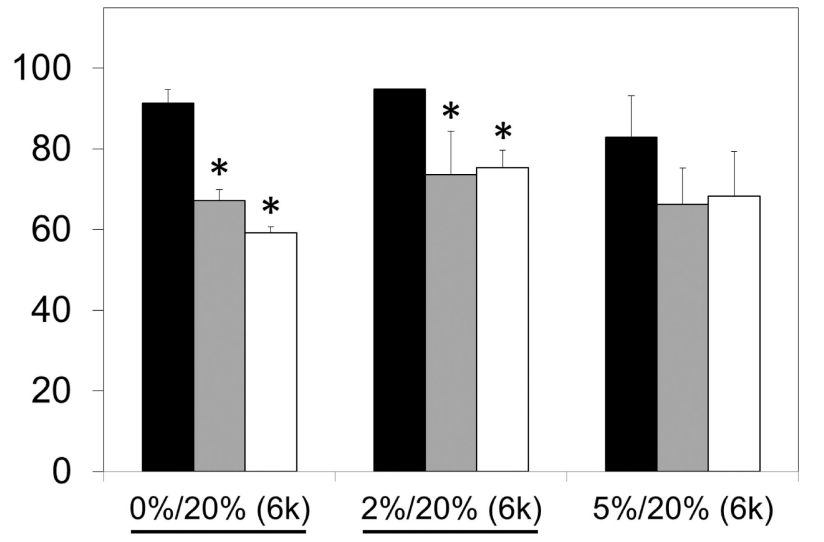

Formulation

Maximum strain of agarose and PEG-DA IPNs encapsulated with chondrocytes at 0 and 3 weeks. A total of 9 groups are compared three at a time, grouped as (a) pure $20 \%$ PEG-DA gels with varied molecular weights of 2000 (2k), 3400 (3.4k) and 6000 (6k) Da, (b) IPNs varying molecular weights, (c) IPNs with varying concentrations of PEG-DA, and (d) IPNs with varying agarose concentrations. As with maximum stress (Figure 8), cell-laden gels appeared to have a limit to the amount of strain they could withstand, regardless of the performance of their acellular counterparts. Formulations listed as agarose concentration/ PEG-DA concentration (PEG-DA molecular weight), and underlines note the same formulation was shown in a previous panel. Values from acellular gels (taken from Fig. 1b) included for comparison. *Values significantly different from acellular gels with the same formulation $(\mathrm{p}<0.05)$, \#significant differences among groups at the same time point within 

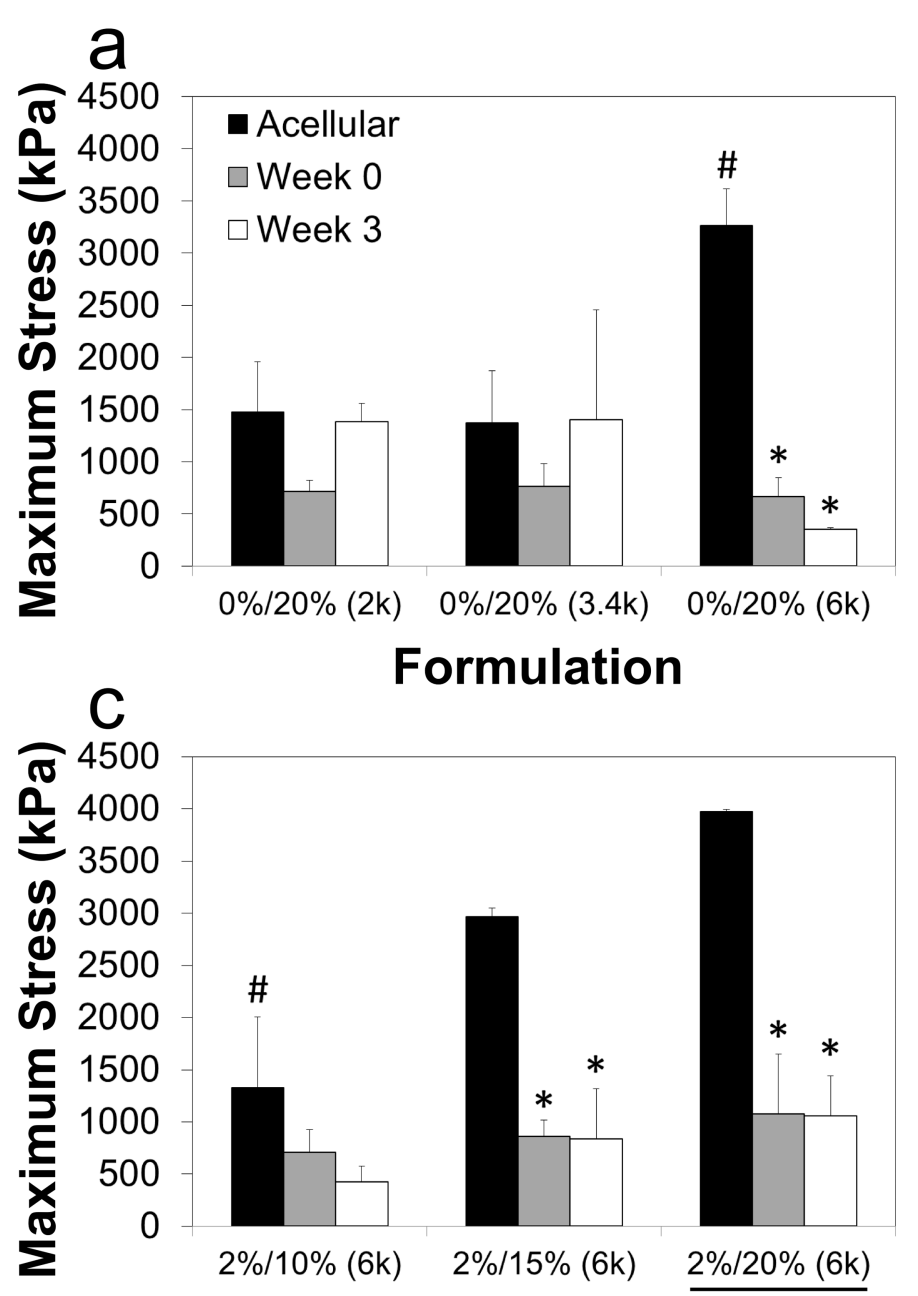

Formulation
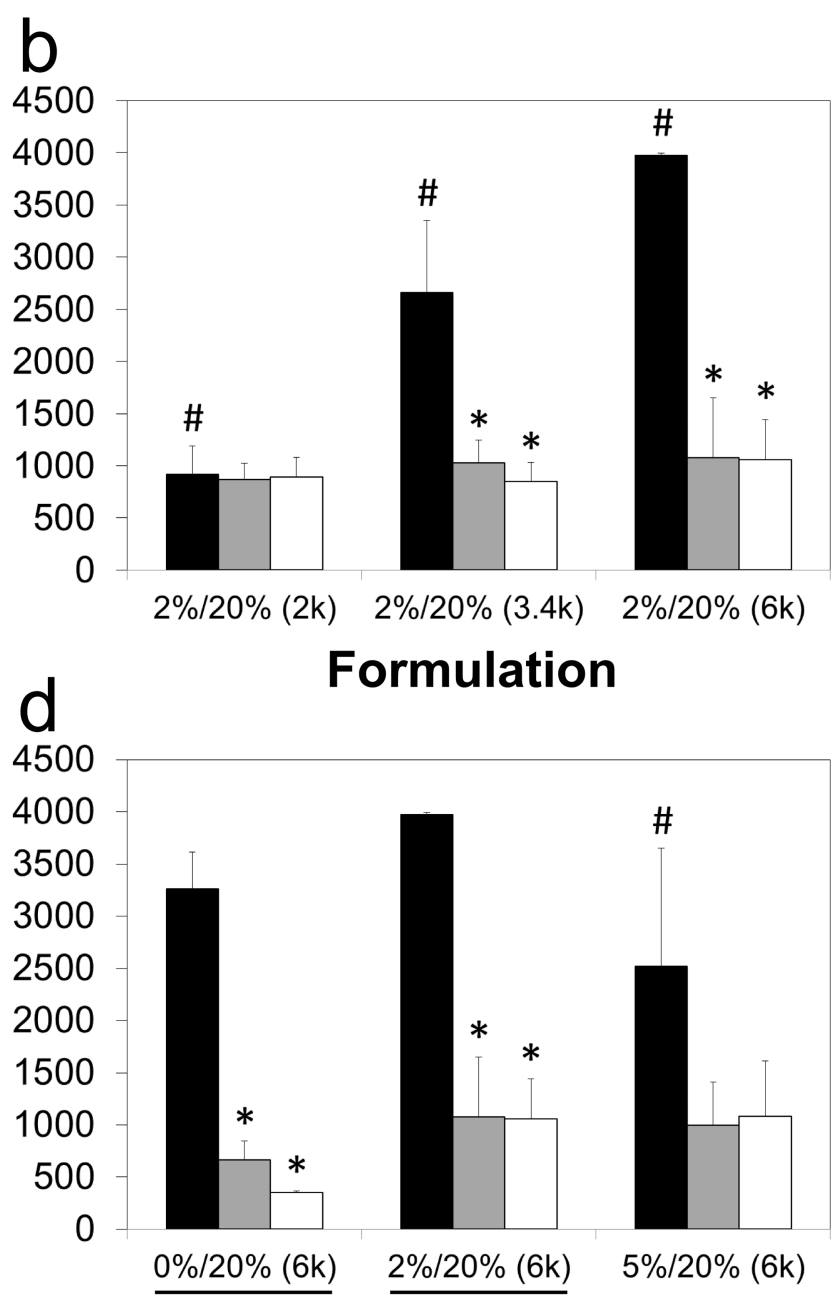

Formulation

Figure 10.

Maximum stress of agarose and PEG-DA IPNs encapsulated with chondrocytes at 0 and 3 weeks. A total of 9 groups are compared three at a time, grouped as (a) pure $20 \%$ PEG-DA gels with varied molecular weights of 2000 (2k), 3400 (3.4k) and 6000 (6k) Da, (b) IPNs varying molecular weights, (c) IPNs with varying concentrations of PEG-DA, and (d) IPNs with varying agarose concentrations. Unlike the shear and compressive moduli, values of the cell-encapsulated gels did not follow the same trends as their acellular counterparts due to an inability to withstand a stress of more than approximately $1500 \mathrm{kPa}$. Formulations listed as agarose concentration/PEG-DA concentration (PEG-DA molecular weight), and underlines note the same formulation was shown in a previous panel. Values from acellular gels (taken from Fig. 1a) included for comparison. *Values significantly different from acellular gels with the same formulation $(\mathrm{p}<0.05)$, \#significant differences among groups at the same time point within the same panel $(\mathrm{p}<0.05)$. 


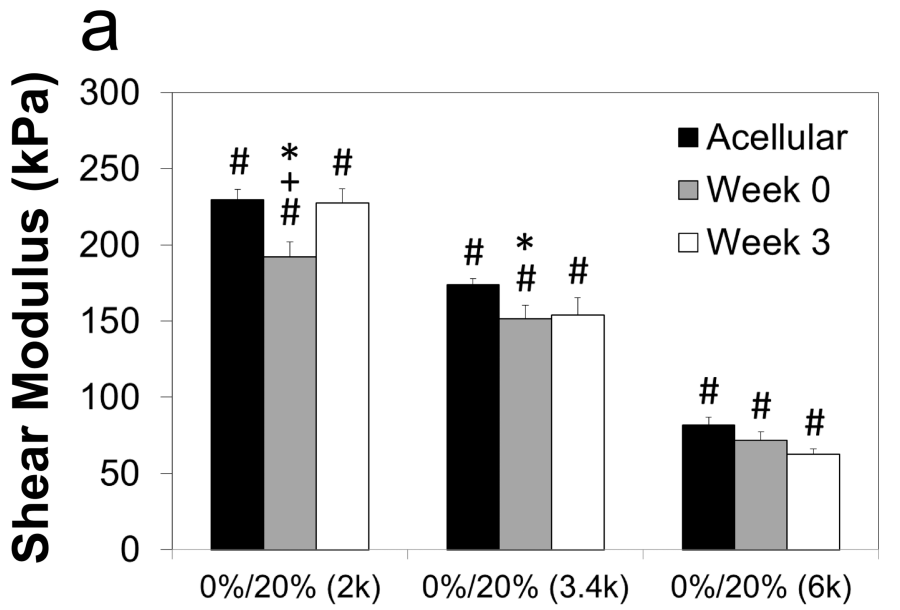

Formulation

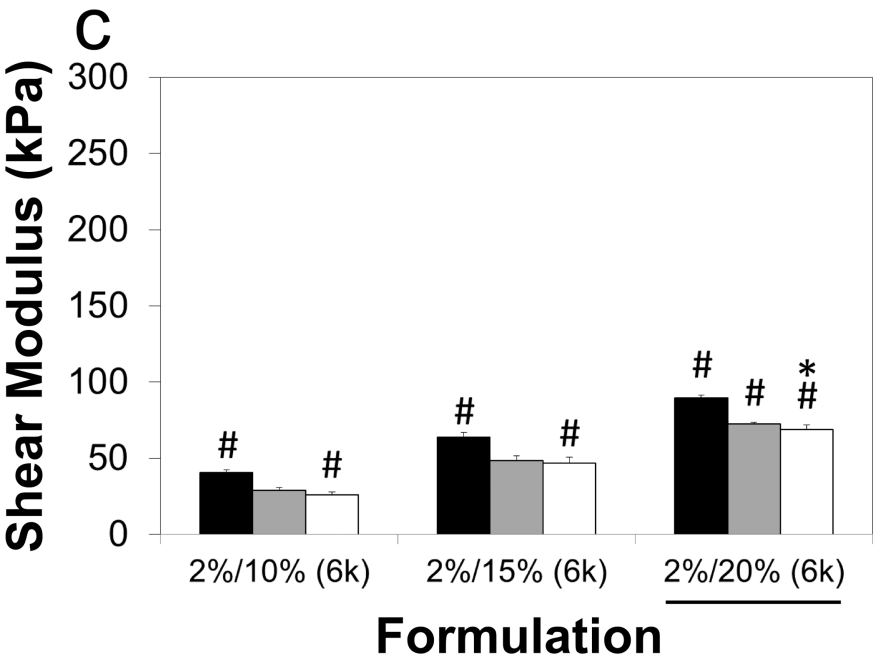

b

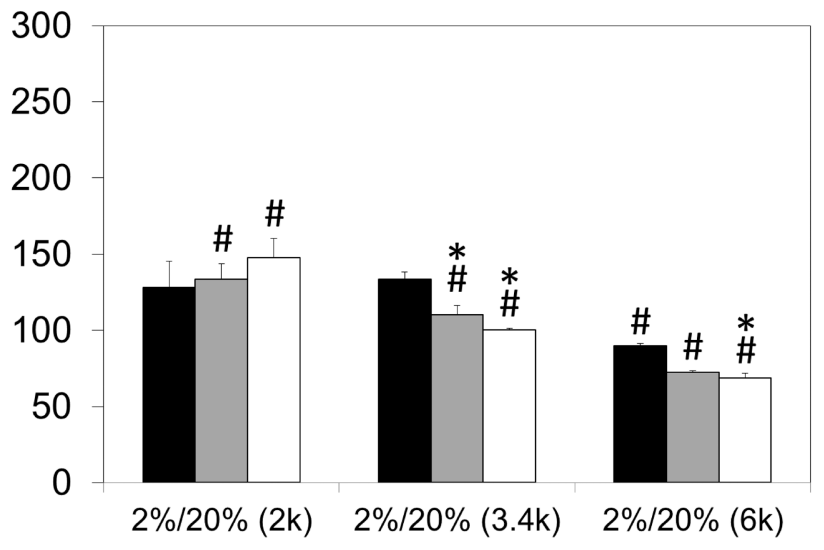

Formulation

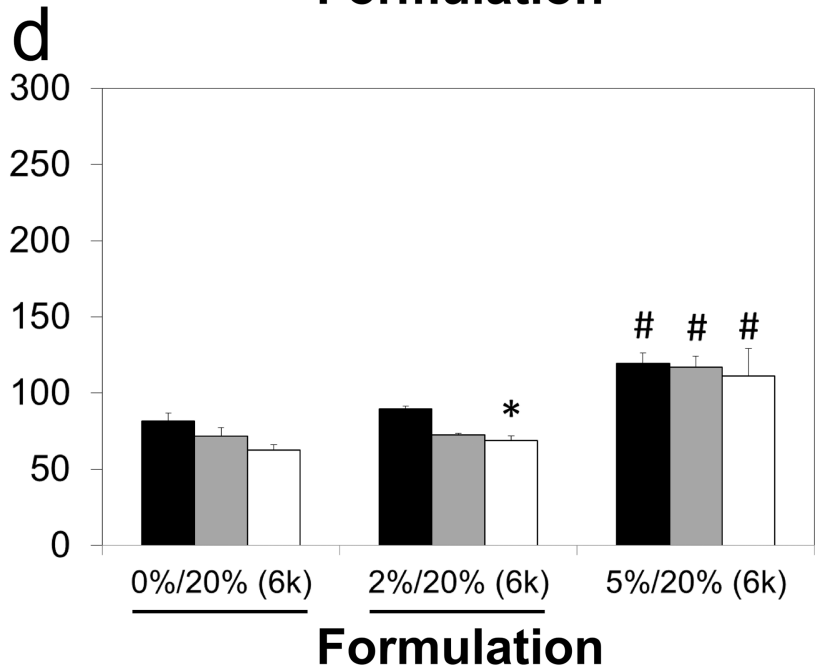

Figure 11.

Shear moduli of agarose and PEG-DA IPNs encapsulated with chondrocytes at 0 and 3 weeks. A total of 9 groups are compared three at a time, grouped as (a) pure 20\% PEG-DA gels with varied molecular weights of 2000 (2k), 3400 (3.4k) and 6000 (6k) Da, (b) IPNs varying molecular weights, (c) IPNs with varying concentrations of PEG-DA, and (d) IPNs with varying agarose concentrations. Cell-laden gels typically followed the same trends as acellular gels, with little difference between time points. Formulations listed as agarose concentration/PEG-DA concentration (PEG-DA molecular weight), and underlines note the same formulation was shown in a previous panel. Values from acellular gels (taken from Fig. 2a) included for comparison. *Values significantly different from acellular gels with the same formulation $(\mathrm{p}<0.05)$, \#significant differences among groups at the same time point within the same panel $(\mathrm{p}<0.05)$, +significant differences between week 0 and week 3 for a single group $(\mathrm{p}<0.05)$. 


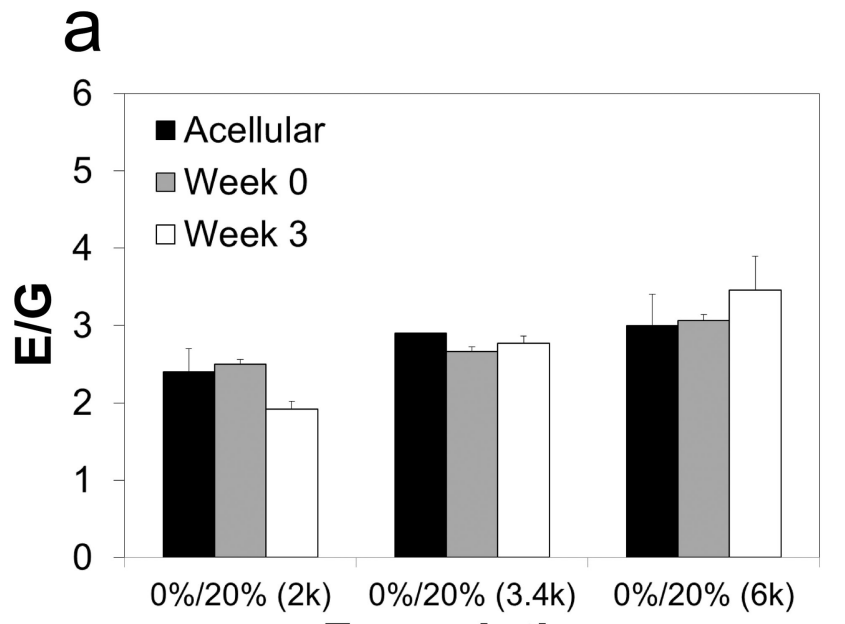

Formulation

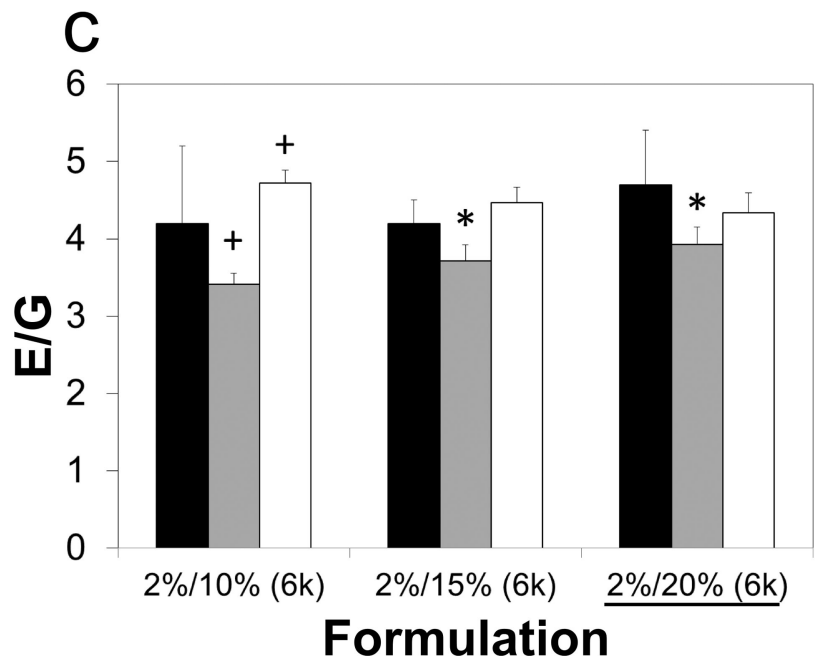

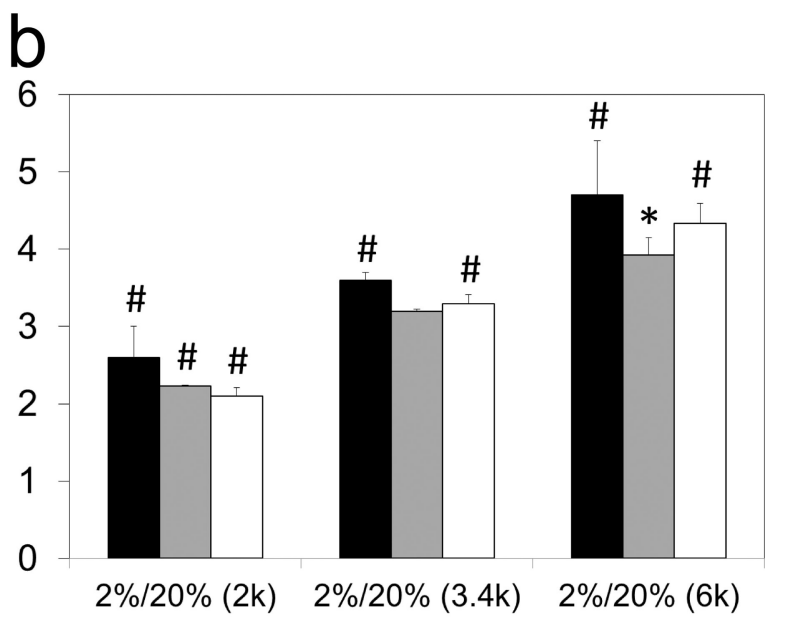

Formulation

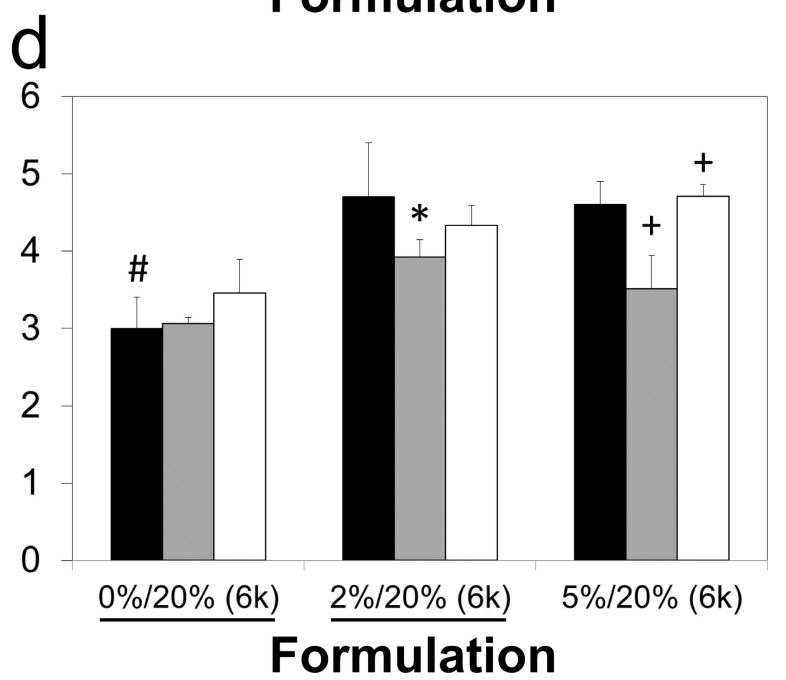

Figure 12.

Ratio of compressive modulus to shear modulus (E/G) of agarose and PEG-DA IPNs encapsulated with chondrocytes at 0 and 3 weeks. A total of 9 groups are compared three at a time, grouped as (a) pure 20\% PEG-DA gels with varied molecular weights of 2000 (2k), $3400(3.4 \mathrm{k})$ and 6000 (6k) Da, (b) IPNs varying molecular weights, (c) IPNs with varying concentrations of PEG-DA, and (d) IPNs with varying agarose concentrations. Very few significant differences were found in ideal elastic behavior between cell-laden and acellular gels. Formulations listed as agarose concentration/PEG-DA concentration (PEG-DA molecular weight), and underlines note the same formulation was shown in a previous panel. Values from acellular gels (taken from Fig. 2b) included for comparison. *Values significantly different from acellular gels with the same formulation $(p<0.05)$, \#significant differences among groups at the same time point within the same panel $(\mathrm{p}<0.05)$, + significant differences between week 0 and week 3 for a single group $(\mathrm{p}<0.05)$. 
Table 1

Formulations of hydrogels selected for cell encapsulation

\begin{tabular}{llll}
\hline Formulation & $\begin{array}{l}\text { Agarose } \\
\text { Concentration } \\
(\mathbf{w} / \mathbf{v} \%)\end{array}$ & $\begin{array}{l}\text { PEG-DA } \\
\text { Concentration } \\
(\mathbf{w} / \mathbf{v} \%)\end{array}$ & $\begin{array}{l}\text { PEG-DA } \\
\text { Molecular } \\
\text { Weight }\end{array}$ \\
$0 \% / 20 \%(2 \mathrm{k})$ & 0 & 20 & 2000 \\
$0 \% / 20 \%(3.4 \mathrm{k})$ & 0 & 20 & 3400 \\
$0 \% / 20 \%(6 \mathrm{k})$ & 0 & 20 & 6000 \\
$2 \% / 20 \%(2 \mathrm{k})$ & 2 & 20 & 2000 \\
$2 \% / 20 \%(3.4 \mathrm{k})$ & 2 & 20 & 3400 \\
$2 \% / 20 \%(6 \mathrm{k})$ & 2 & 20 & 6000 \\
$2 \% / 15 \%(6 \mathrm{k})$ & 2 & 15 & 6000 \\
$2 \% / 10 \%(6 \mathrm{k})$ & 2 & 10 & 6000 \\
$5 \% / 20 \%(6 \mathrm{k})$ & 5 & 20 & 6000 \\
\hline
\end{tabular}

Indicates formulation was analyzed for glycosaminoglycan (GAG) and DNA content. 
Table 2

Summary of the most significant factors that influence each property

\begin{tabular}{lll}
\hline Property & Most Significant Factor & Relationship \\
Shear Modulus (G) & PEG-DA concentration & Shear moduli were higher with greater PEG-DA concentration \\
Compressive Modulus (E) & PEG-DA concentration & Compressive moduli were higher with greater PEG-DA concentration \\
Mass swelling ratio (q) & PEG-DA concentration & Mass swelling ratios were lower with greater PEG-DA concentration \\
PEG-DA content & PEG-DA concentration & PEG-DA content was higher with greater PEG-DA concentration $b$ \\
Mesh size $(\xi)$ & PEG-DA concentration & Mesh sizes were lower with greater PEG-DA concentration \\
Crosslink density $\left(\rho_{\mathrm{x}}\right)$ & PEG-DA concentration & Crosslink densities were higher with greater PEG-DA concentration \\
E/G & PEG-DA molecular weight ${ }^{a}$ & E/G ratios were higher with higher PEG-DA molecular weight \\
Maximum stress & PEG-DA molecular weight & Maximum stresses were higher with higher PEG-DA molecular weight \\
Maximum strain & PEG-DA molecular weight & Maximum strains were higher with higher PEG-DA molecular weight \\
\hline
\end{tabular}

${ }^{a}$ Agarose concentration also strongly influenced E/G ratios, with an increase in agarose concentration leading to higher E/G ratios.

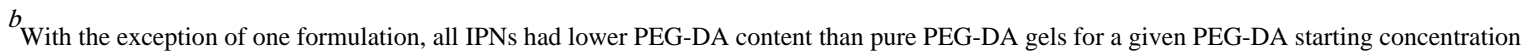
$(\mathrm{p}<0.05)$. 